\title{
Effect of Hot Water and Chitosan Treatments for Improving the Quality and Increasing Storability of Globe Artichoke.
}

\author{
Sheren A. Atala ${ }^{1}$; Saad M. EL-M. ${ }^{1}$ and Saleh M. A. ${ }^{1}$ \\ Postharvest vegetables Dep. Horti. Res. Inst., ARC, Giza, Egypt. \\ * Corresponding author: mohsenyoukasha@yahoo.com
}

\begin{abstract}
Artichoke heads of the cultivar "French Hyrious" were harvested at the suitable maturity stage of marketing on $5^{\text {th }}$ and $9^{\text {th }}$ of February in 2017 and 2018 season, respectively from private farm in Kafr El Dawar Sidi Ghazi - Behera Governorate, Egypt and shifted to the laboratory of Vegetable Handling Research Department, Horticultural Research Institute, Agricultural Research Center, to evaluate the effects of hot water and chitosan treatments, alone or in combination as postharvest treatment on quality attributes of artichoke head during cold storage. Results showed that all postharvest treatments of artichoke heads slowed the rate of weight loss, had the highest compactness and gave the highest value of $\mathrm{L}$ and hue angle values, resulted in lighter color and inhibit the loss of green color comparatively to the highest ones obtained from untreated control. Artichoke heads dipped in hot water at $44^{\circ} \mathrm{C}$ then chitosan $1 \%$ or hot water at $44^{\circ} \mathrm{C}$ then chitosan $1.5 \%$ were perceived to have the highest intensities of freshness, greenness and compactness. Artichoke heads dipped in hot water at $44^{\circ}$ $\mathrm{C}$ then chitosan $1 \%$ produced an improvement in market quality; it would reduce weight loss percentage, color change and maintained ascorbic acid content, TSS, dry matter and inulin content and did not show any change in general appearance after 28 days of storage at $0{ }^{\circ} \mathrm{C}$ and $95 \% \mathrm{RH}$.
\end{abstract}

Key words: artichoke heads, hot water, chitosan, quality, cold storage.

\section{Introduction}

Globe artichoke (Cynara scolymus L.) is considered as one of the important vegetable crops for local consumption and export in Egypt. The nutraceutical properties of artichokes are mainly due to its high polyphenolic content, which can be up to $2 \%$ of the fresh weight (Mileo et al., 2012), and the presence of fructan inulin that has been reported to have a prebiotic function that stimulates the growth of intestinal bifidobacteria (Lavermicocca et al., 2016).

However, artichoke is a highly perishable commodity, characterized by rapid rates of respiration and water loss (Kader, 1992). The main factors of artichoke heads quality degradation during prolonged storage are mostly due to decay development shriveling, associated with rapid water loss, poor external appearance, as well as reduced sensory quality. Therefore, some postharvest treatments in addition to refrigeration have been proposed for use in maintaining quality and improving storability of artichoke heads i.e., treatment with chitosan, hot water and combined between them.

Chitosan, a deacetylated derivate of chitin, is a high molecular weight cationic linear polysaccharide composed of d- glucosamine and, to a lesser extent, $\mathrm{N}$ - acetyl- d- glucosamine with a $B-1,4-$ linkage ( Rinaudo, 2006). Chitosan is typically extracted from an abundant source of shellfish exoskeletons or the cell wall of some microorganisms and fungi (Hirano et al., 1976). Chitosan coatings are considered the best edible and biologically safe preservative coatings for different types of vegetables and fruits, with functional advantages; where chitosan coating acts as a semipermeable barrier against oxygen, carbon dioxide and moisture, thereby reduced respiration rates (Vargas et al., 2006), water loss (Zahoorullah et al., 2017), maintained the quality (Ardakani and Mostofi, 2019), extended storage periods(Youssef et al., 2015 and Ahmed et al., 2016), reduce color changes (Suseno et al., 2014) and controlled microbial growth (Mahmoud et al., 2017).

Heat treatment is an effective non-chemical method for controlling postharvest pests and diseases if suitable combinations of temperatures and exposure times are selected to prevent quality loss. The use of hot water treatment is an accurate method to reduce weight loss, decay percentage, visual appearance, compactness, brown discoloration, TSS, ascorbic acid, dry matter and inulin sugar of artichoke (Atala, 2006) maintain fruit quality (Fallik, 2004; Abdalla, 2013) inhibit color development and extend shelf life (Gonzalez-Aguilar et al., 1999).

The use of a combination of such techniques in the postharvest handling of fresh products is increasing. There are only a few reports about the effects of an integrated treatment involving heat treatment and chitosan coating on the fruit during storage. Djioua et al. (2010) found that the chitosan coating combined with hot water treatment improved the antimicrobial effect on fresh-cut mangoes. The combined effect of chitosan and hot water, Zhao et al. (2009) found that the heat treatment plus chitosan treated apple fruit showed the lowest respiration rate, ethylene evolution, malondialdehyde and membrane 
leakage, and consumer acceptance. At the same time, this combined treatment could inhibit the lost of green color and weight loss compared with hot water alone (Shao et al., 2012). Also, Chailoo and Asghari (2011) noticed that combined treatment with $0.5 \%$ chitosan and hot water at $50{ }^{\circ} \mathrm{C}$ after 60 days of storage life showed good result in compartion to untreated control.

The present study investigated the effects of hot water and chitosan treatments, alone or in combination on quality attributes of artichoke head during cold storage.

\section{Materials and methods}

Artichoke marketable heads of the cultivar "French Hyrious" were harvested at the suitable maturity stage of marketing with a dimension of (7$12 \mathrm{~cm}$ ) on $5^{\text {th }}$ and $9^{\text {th }}$ of February in 2017 and 2018 season respectively from private farm in Kafr El Dawar - Sidi Ghazi - Behera Governorate, Egypt and shifted to the laboratory of Vegetable Handling Research Department, Horticultural Research Institute, Agricultural Research Center, Ministry of Agriculture, A.R.E. Heads were cleaned with dry towels, graded and free from blemishes were selected for storage experiment.

Artichoke heads were dipped in hot water and chitosan alone or in combination between them as follow hot water at $44^{\circ} \mathrm{C}$ for 5 minutes, hot water at $48^{\circ} \mathrm{C}$ for 2 minutes, solution of chitosan at $1 \%$ for 5 min., solution of chitosan at $1.5 \%$ for $5 \mathrm{~min}$., hot water at $44^{\circ} \mathrm{C}$ then chitosan at $1 \%$, hot water at $44^{\circ} \mathrm{C}$ then chitosan at $1.5 \%$, hot water at $48^{\circ} \mathrm{C}$ then chitosan at $1 \%$, hot water at $48^{\circ} \mathrm{C}$ then chitosan at $1.5 \%$ and untreated control ( dipping in distilled water for $5 \mathrm{~min}$.).

All samples of artichoke heads were air dried, the artichoke heads from each treatment were packed in polypropylene bags $(20 \times 30 \mathrm{~cm})$ of $30 \mu \mathrm{m}$ thickness and each had three artichoke heads represented as one replicate and then heat sealed. Twelve replicates were prepared for each treatment. The samples were arranged in complete randomized design with three replicates and stored at $0{ }^{\circ} \mathrm{C}$ and 95\% RH for 28 days. Three replicates from each treatment were examined immediately after harvest and every 7 days intervals for the following properties.

Loss in weight percentage calculated by the following equation:

Loss in weight $\%=$ Initial weight of head weight of head at sampling date / the initial weight of the head X100. The general appearance: as evaluated using a scale from 9 to 1 , where $9=$ excellent, $7=$ good, $5=$ fair, $3=$ poor and $1=$ unsalable heads rating (5) or below were considered as unmarketable, as described by Kader et al. (1973). The compactness: Score rating from 5 to 1 , where $5=$ tight, $4=$ few basal bracts pointing a way from rather than toward tip of bud, $3=$ several whorls of bracts pointing a way from rather than toward tip of bud, $2=$ all or most outer bracts open, $1=$ all outer and more antrally located bracts open. External surface color was evaluated by a color meter (Minolta CR 200) to measure the lightness ( $\mathrm{L}$ value) and hue angle $\left(\mathrm{h}^{\circ}\right)$ value. Dry matter: The percentage of dry matter content was determined in $100 \mathrm{~g}$. of fresh heads which were dried in an oven at $70^{\circ} \mathrm{C}$ till constant pitting was reached, the results were calculated as $\mathrm{g} /$ $100 \mathrm{~g}$. fresh weight. Ascorbic acid content (as indicated for vit. C). It was determined (as fresh samples of heads) by titration method using 2, 6 discloro phenol indophenols as described in (Ranganna, 1979).Total soluble solids percentage (TSS) \% it was measured from the fresh materials using PR-101 digital refractometer. Inulin sugar: Inulin was determined in flower heads according to the method of Winton and Winton (1958).

\section{Statistical analysis}

Data were statistically analyzed using the analysis of variance described by Snedecor and Cochran (1980). The method of Duncan multiple range test was applied for than comparison between means according to Waller and Duncan (1969).

\section{Results and Discussion}

\section{Weight loss percentage}

Data in Table (1) showed that weight loss percentage increased with extending cold storage period. Highest losses of weight were obtained at the end of storage period (Mohamedien et al., 2019). Normally, the weight loss occurs during the fruit storage due to respiratory process, the transference of humidity and some processes of oxidation (Wills et al., 1998).

Concerning the effect of postharvest treatments, data revealed that there were significant differences among treatments in weight loss percentage during storage. All postharvest treatments retained their weight during storage as compared with untreated control. Moreover, artichoke heads dipped in hot water at $44^{\circ} \mathrm{C}$ plus chitosan $1 \%$ was the most effective treatment in reducing weight loss $\%$ followed by hot water at $44^{\circ} \mathrm{C}$ plus chitosan $1.5 \%$ with significant differences between them in the two seasons followed by chitosan at $1 \%$ or $1.5 \%$ alone. Hot water at $48^{\circ} \mathrm{C}$ was less effective in this concern. These results were achieved in the two seasons and were in agreement with Xu et al. (2007) for chitosan; Fallik (2004) for hot water and Ramos-García et al. (2009) for hot water and chitosan. This positive effect of edible coatings is based on their hygroscopic properties, which enables the formation of a water barrier between the fruit and the environment, thus reducing weight loss. In addition, the decreasing of weight loss may be attributed to controlling of decay and its damages through the 
biological activity of chitosan against postharvest diseases and make a thin film surrounding the fruit peel and induced a modification of atmosphere around the fruits.

Table 1. Effect of hot water treatment and chitosan coating alone or in a combination between them on weight loss percentage of artichoke heads during cold storage.

\begin{tabular}{|c|c|c|c|c|c|c|}
\hline \multirow{3}{*}{ Treatments } & \multicolumn{5}{|c|}{ Storage period(days) } & \multirow{3}{*}{ Mean } \\
\hline & \multicolumn{5}{|c|}{2017 season } & \\
\hline & start & 7days & 14days & 21days & 28days & \\
\hline Chitosan 1\% & $0.00 \mathrm{Z}$ & $0.07 \mathrm{X}-\mathrm{Z}$ & $0.21 \mathrm{~T}-\mathrm{W}$ & $0.57 \mathrm{O}$ & $0.79 \mathrm{LM}$ & $0.33 \mathrm{G}$ \\
\hline Chitosan $1.5 \%$ & $0.00 \mathrm{Z}$ & $0.09 \mathrm{~W}-\mathrm{Z}$ & $0.26 \mathrm{~S}-\mathrm{U}$ & $0.72 \mathrm{MN}$ & $0.98 \mathrm{JK}$ & $0.41 \mathrm{~F}$ \\
\hline Hot water $44^{\circ} \mathrm{C}$ & $0.00 \mathrm{Z}$ & $0.13 \mathrm{~V}-\mathrm{Y}$ & $0.41 \mathrm{PQ}$ & $0.90 \mathrm{~K}$ & $1.23 \mathrm{GH}$ & $0.53 \mathrm{E}$ \\
\hline Hot water $44^{\circ} \mathrm{C}+$ Chitosan $1 \%$ & $0.00 \mathrm{Z}$ & $0.03 \mathrm{YZ}$ & $0.09 \mathrm{~W}-\mathrm{Z}$ & $0.24 \mathrm{~S}-\mathrm{V}$ & $0.40 \mathrm{P}-\mathrm{R}$ & 0.15 I \\
\hline Hot water $44^{\circ} \mathrm{C}+$ Chitosan $1.5 \%$ & $0.00 \mathrm{Z}$ & $0.06 \mathrm{X}-\mathrm{Z}$ & $0.14 \mathrm{~V}-\mathrm{Y}$ & $0.33 \mathrm{Q}-\mathrm{S}$ & $0.62 \mathrm{NO}$ & $0.23 \mathrm{H}$ \\
\hline Hot water $48^{\circ} \mathrm{C}$ & $0.00 \mathrm{Z}$ & $0.45 \mathrm{P}$ & $1.21 \mathrm{GH}$ & $1.67 \mathrm{~F}$ & $2.57 \mathrm{C}$ & $1.18 \mathrm{~B}$ \\
\hline Hot water $48^{\circ} \mathrm{C}+$ Chitosan $1 \%$ & $0.00 \mathrm{Z}$ & $0.16 \mathrm{U}-\mathrm{X}$ & $0.62 \mathrm{NO}$ & $1.16 \mathrm{HI}$ & $1.76 \mathrm{~F}$ & $0.74 \mathrm{D}$ \\
\hline Hot water $48^{\circ} \mathrm{C}+$ Chitosan $1.5 \%$ & $0.00 \mathrm{Z}$ & $0.29 \mathrm{R}-\mathrm{T}$ & $0.87 \mathrm{KL}$ & $1.30 \mathrm{G}$ & $2.01 \mathrm{E}$ & $0.89 \mathrm{C}$ \\
\hline Control & $0.00 \mathrm{Z}$ & $1.07 \mathrm{IJ}$ & $2.13 \mathrm{D}$ & $3.62 \mathrm{~B}$ & $4.54 \mathrm{~A}$ & $2.27 \mathrm{~A}$ \\
\hline Mean & $0.00 \mathrm{E}$ & $0.26 \mathrm{D}$ & $0.66 \mathrm{C}$ & $1.17 \mathrm{~B}$ & $1.65 \mathrm{~A}$ & \\
\hline \multicolumn{7}{|c|}{2018 season } \\
\hline Chitosan 1\% & $0.00 \mathrm{Z}$ & $0.18 \mathrm{WX}$ & $0.36 \mathrm{ST}$ & $0.73 \mathrm{O}$ & $0.96 \mathrm{M}$ & $0.44 \mathrm{G}$ \\
\hline Chitosan $1.5 \%$ & $0.00 \mathrm{Z}$ & $0.22 \mathrm{~W}$ & $0.43 \mathrm{R}$ & $0.92 \mathrm{MN}$ & $1.17 \mathrm{KL}$ & $0.55 \mathrm{~F}$ \\
\hline Hot water $44^{\circ} \mathrm{C}$ & $0.00 \mathrm{Z}$ & $0.27 \mathrm{~V}$ & $0.62 \mathrm{P}$ & $1.13 \mathrm{~L}$ & $1.46 \mathrm{I}$ & $0.70 \mathrm{E}$ \\
\hline Hot water $44^{\circ} \mathrm{C}+$ Chitosan $1 \%$ & $0.00 \mathrm{Z}$ & $0.11 \mathrm{Y}$ & $0.30 \mathrm{UV}$ & $0.49 \mathrm{Q}$ & $0.62 \mathrm{P}$ & $0.30 \mathrm{I}$ \\
\hline Hot water $44^{\circ} \mathrm{C}+$ Chitosan $1.5 \%$ & $0.00 \mathrm{Z}$ & $0.13 \mathrm{XY}$ & $0.34 \mathrm{TU}$ & $0.60 \mathrm{P}$ & $0.88 \mathrm{~N}$ & $0.39 \mathrm{H}$ \\
\hline Hot water $48^{\circ} \mathrm{C}$ & $0.00 \mathrm{Z}$ & $0.53 \mathrm{Q}$ & $1.44 \mathrm{IJ}$ & $1.96 \mathrm{G}$ & $2.87 \mathrm{C}$ & $1.36 \mathrm{~B}$ \\
\hline Hot water $48^{\circ} \mathrm{C}+$ Chitosan $1 \%$ & $0.00 \mathrm{Z}$ & $0.33 \mathrm{TU}$ & $0.91 \mathrm{MN}$ & $1.39 \mathrm{~J}$ & $2.03 \mathrm{~F}$ & $0.93 \mathrm{D}$ \\
\hline Hot water $48^{\circ} \mathrm{C}+$ Chitosan $1.5 \%$ & $0.00 \mathrm{Z}$ & $0.41 \mathrm{RS}$ & $1.16 \mathrm{KL}$ & $1.58 \mathrm{H}$ & $2.34 \mathrm{E}$ & $1.10 \mathrm{C}$ \\
\hline Control & $0.00 \mathrm{Z}$ & $1.19 \mathrm{~K}$ & $2.45 \mathrm{D}$ & $3.95 \mathrm{~B}$ & $4.94 \mathrm{~A}$ & $2.51 \mathrm{~A}$ \\
\hline Mean & $0.00 \mathrm{E}$ & $0.37 \mathrm{D}$ & $0.89 \mathrm{C}$ & $1.42 \mathrm{~B}$ & $1.92 \mathrm{~A}$ & \\
\hline
\end{tabular}

Means in the same column having the same letter are not significantly different at 0.05 level by Duncan's multiple rang test.

Minimizing weight loss from hot water treatment during storage may be due to heat treatments inhibited the ripening of many fruits and vegetables. Other workers reported that hot water treatments appeared to inhibit certain ripening processes as indicated by the relatively low respiration rats and ethylene evolution of hot water treated fruit (Fallik, 2004) which in turn reduce weight loss.

In general, the interaction between postharvest treatments and storage periods was significant effect on weight loss percentage in the two seasons. After 28 days of storage, the lowest value of weight loss was recorded from heads treated with hot water at $44^{\circ} \mathrm{C}$ plus chitosan $1 \%$ while the highest ones were obtained from untreated control. These results agreement with those obtained by Djioua et al. (2010) and Chailoo and Asghari (2011) found that the combination of hot water and chitosan treatments as barriers offers a greater potential for shelf life extension on processed vegetables.

The favorable effect of chitosan treatment in reducing weight loss may be due to the formation of thin layer covering the fruit, which prevents moisture loss, reduces gas exchange and subsequently inhibits metabolic activities, resulting in lowering weight loss (Raymond et al., 2012).

Lowest weight loss from combined between chitosan and hot water treatments during storage may be due to chitosan film after the hot water showed an advantage effect to control the changes of $\mathrm{CO}_{2} / \mathrm{O}_{2}$ (Gemma and Du 1998). This permselectivity film could not lead to the endogenous anaerobic conditions and damage and help for the storage of heat-treated apple fruits. Thus, combined treatment alleviates the positive impacts (lower respiration rate for ethylene evolution, membrane leakage and sustained firmness) of chitosan coating (RamosGarcía et al., 2009).

\section{General appearance (GA)}

Data in Table (2) show that general appearance (score) of artichoke heads was decreased with the prolongation of storage period. These results were in agreement with those obtained by (Mohamedien et al., 2019). The decreases in GA of artichoke heads during storage might be due to shriveling, color change and decay (Nobile et al., 2009).

Concerning the effect of postharvest treatments, data revealed that there were significant differences between postharvest treatments and untreated control during storage. Artichoke heads treated with all postharvest treatments had significantly the highest score of appearance as compared with untreated control. However, artichoke heads dipped in hot water at $44^{\circ} \mathrm{C}$ plus chitosan $1 \%$ and hot water at $44^{\circ}$ $\mathrm{C}+$ chitosan $1.5 \%$ were the most effective treatments for maintaining general appearance with no significant differences between them followed by 
chitosan $1 \%$ or $1.5 \%$ alone. Hot water at $48^{\circ} \mathrm{C}$ alone or combination with chitosan $1 \%$ or $1.5 \%$ were less effective treatments in maintaining GA, while untreated control recorded the lowest ones in this concern. These results were achieved in the two seasons and were in agreement with (HernandezMunoz et al., 2008; Ardakani \& Mostofi, 2019 for chitosan, Mama et al. 2016 for hot water, RamosGarcia et al. 2009 for chitosan and hot water).The decreasing of GA may be due to chitosan coating acts as a semipermeable barrier on the surface of fruit and vegetables against oxygen, carbon dioxide and moisture, thereby reducing respiration, water loss, respiratory activity and degradation by enzymes and microbial rot of fruits, counteracting the dehydration and shrinkage of the fruit, and ethylene production and maintaining the overall quality (Velickova et al., 2013). Chitosan coatings can also improve the sensory attributes such as appearance and color during the low-temperature storage time (Ardakani \& Mostofi, 2019).

Table 2. Effect of hot water treatment and chitosan coating alone or in a combination between them on general appearance (score) of artichoke heads during cold storage.

\begin{tabular}{|c|c|c|c|c|c|c|}
\hline \multirow{3}{*}{ Treatments } & \multicolumn{5}{|c|}{ Storage period(days) } & \multirow{3}{*}{ Mean } \\
\hline & \multicolumn{5}{|c|}{2017 season } & \\
\hline & start & 7days & 14 days & 21 days & 28 days & \\
\hline Chitosan 1\% & $9.00 \mathrm{~A}$ & $9.00 \mathrm{~A}$ & $9.00 \mathrm{~A}$ & $7.67 \mathrm{~A}-\mathrm{C}$ & $6.33 \mathrm{C}-\mathrm{E}$ & $8.20 \mathrm{~B}$ \\
\hline Chitosan $1.5 \%$ & $9.00 \mathrm{~A}$ & $9.00 \mathrm{~A}$ & $9.00 \mathrm{~A}$ & 7.00 B-D & $5.67 \mathrm{D}-\mathrm{F}$ & $7.93 \mathrm{BC}$ \\
\hline Hot water $44^{\circ} \mathrm{C}$ & $9.00 \mathrm{~A}$ & $9.00 \mathrm{~A}$ & $7.67 \mathrm{~A}-\mathrm{C}$ & 6.33 C-E & $5.00 \mathrm{EFG}$ & 7.40 CD \\
\hline Hot water $44^{\circ} \mathrm{C}+$ Chitosan $1 \%$ & $9.00 \mathrm{~A}$ & $9.00 \mathrm{~A}$ & $9.00 \mathrm{~A}$ & $9.00 \mathrm{~A}$ & $8.33 \mathrm{AB}$ & $8.87 \mathrm{~A}$ \\
\hline Hot water $44^{\circ} \mathrm{C}+$ Chitosan $1.5 \%$ & $9.00 \mathrm{~A}$ & $9.00 \mathrm{~A}$ & $9.00 \mathrm{~A}$ & $8.33 \mathrm{AB}$ & 7.00 B-D & 8.47 AB \\
\hline Hot water $48^{\circ} \mathrm{C}$ & $9.00 \mathrm{~A}$ & $9.00 \mathrm{~A}$ & $6.33 \mathrm{C}-\mathrm{E}$ & $4.33 \mathrm{~F}-\mathrm{H}$ & $3.00 \mathrm{HI}$ & $6.33 \mathrm{E}$ \\
\hline Hot water $48^{\circ} \mathrm{C}+$ Chitosan $1 \%$ & $9.00 \mathrm{~A}$ & $9.00 \mathrm{~A}$ & 7.00 B-D & 5.67 D-F & $3.67 \mathrm{GH}$ & 6.87 DE \\
\hline Hot water $48^{\circ} \mathrm{C}+$ Chitosan $1.5 \%$ & $9.00 \mathrm{~A}$ & $9.00 \mathrm{~A}$ & $7.00 \mathrm{~B}-\mathrm{D}$ & $5.00 \mathrm{E}-\mathrm{G}$ & $3.00 \mathrm{HI}$ & $6.60 \mathrm{E}$ \\
\hline Control & $9.00 \mathrm{~A}$ & $8.33 \mathrm{AB}$ & $6.33 \mathrm{C}-\mathrm{E}$ & $3.00 \mathrm{HI}$ & $1.67 \mathrm{I}$ & $5.67 \mathrm{~F}$ \\
\hline Mean & $9.00 \mathrm{~A}$ & $8.93 \mathrm{~A}$ & $7.82 \mathrm{~B}$ & $6.26 \mathrm{C}$ & 4.85 D & \\
\hline \multicolumn{7}{|c|}{2018 season } \\
\hline Chitosan 1\% & $9.00 \mathrm{~A}$ & $9.00 \mathrm{~A}$ & $9.00 \mathrm{~A}$ & $7.67 \mathrm{BC}$ & $6.33 \mathrm{DE}$ & $8.20 \mathrm{AB}$ \\
\hline Chitosan $1.5 \%$ & $9.00 \mathrm{~A}$ & $9.00 \mathrm{~A}$ & $9.00 \mathrm{~A}$ & 7.00 CD & $5.67 \mathrm{EF}$ & $7.93 \mathrm{BC}$ \\
\hline Hot water $44^{\circ} \mathrm{C}$ & $9.00 \mathrm{~A}$ & $9.00 \mathrm{~A}$ & $8.33 \mathrm{AB}$ & 6.33 DE & $5.00 \mathrm{FG}$ & $7.53 \mathrm{C}$ \\
\hline Hot water $44^{\circ} \mathrm{C}+$ Chitosan $1 \%$ & $9.00 \mathrm{~A}$ & $9.00 \mathrm{~A}$ & $9.00 \mathrm{~A}$ & $8.33 \mathrm{AB}$ & $8.33 \mathrm{AB}$ & $8.73 \mathrm{~A}$ \\
\hline Hot water $44^{\circ} \mathrm{C}+$ Chitosan $1.5 \%$ & $9.00 \mathrm{~A}$ & $9.00 \mathrm{~A}$ & $9.00 \mathrm{~A}$ & $8.33 \mathrm{AB}$ & $7.00 \mathrm{CD}$ & 8.47 AB \\
\hline Hot water $48^{\circ} \mathrm{C}$ & $9.00 \mathrm{~A}$ & $9.00 \mathrm{~A}$ & $6.33 \mathrm{DE}$ & $4.33 \mathrm{GH}$ & $3.00 \mathrm{IJ}$ & 6.33 DE \\
\hline Hot water $\mathbf{4 8}^{\circ} \mathrm{C}+$ Chitosan $1 \%$ & $9.00 \mathrm{~A}$ & $9.00 \mathrm{~A}$ & $7.67 \mathrm{BC}$ & $5.00 \mathrm{FG}$ & $3.67 \mathrm{HI}$ & 6.87 D \\
\hline Hot water $48^{\circ} \mathrm{C}+$ Chitosan $1.5 \%$ & $9.00 \mathrm{~A}$ & $9.00 \mathrm{~A}$ & $7.00 \mathrm{CD}$ & $5.00 \mathrm{FG}$ & $3.67 \mathrm{HI}$ & $6.73 \mathrm{D}$ \\
\hline Control & $9.00 \mathrm{~A}$ & $9.00 \mathrm{~A}$ & $6.33 \mathrm{DE}$ & $3.67 \mathrm{HI}$ & $2.33 \mathrm{~J}$ & $6.07 \mathrm{~F}$ \\
\hline Mean & $9.00 \mathrm{~A}$ & $9.00 \mathrm{~A}$ & $9.00 \mathrm{~A}$ & $7.67 \mathrm{BC}$ & $6.33 \mathrm{DE}$ & \\
\hline
\end{tabular}

Means in the same column having the same letter are not significantly different at 0.05 level by Duncan's multiple rang test.

Hot water maintaining postharvest quality by delayed ripening rate by delaying softening, weight loss and degradation of chlorophyll and carotenoid. Decreasing activity of cell wall degrading enzyme, disorder of the ethylene synthesis enzyme (Mama et al., 2016). A possible mechanism of hot water for the control of decay development is direct inhibition on fungi spores at high temperature (Fallik , 2004).

Chitosan coating and hot water were effective option for maintaining plant quality. It has been well established that a reduction in fruit respiration and ethylene evolution correlates with delayed senescence (Ramos-Garcia et al., 2009).Chitosan film after the hot water showed an advantage effect to control the changes of $\mathrm{Co}_{2} / \mathrm{O}_{2}$ during the whole storage (Gemma and Du 1998).

In general, the interaction between postharvest treatments and storage periods was significant in the two seasons. Results recorded that artichoke heads dipped in hot water at $44^{\circ} \mathrm{C}$ plus chitosan $1 \%$ did not show any changes in GA till the end of storage period . While, hot water at $44^{\circ} \mathrm{C}$ plus chitosan $1.5 \%$ treatment rated good appearance at the same period. Hot water or chitosan alone gave good appearance after 21 days of storage at $0{ }^{\circ} \mathrm{C}$. On the other hand, untreated control had the unsalable appearance after 28 days of storage at $0^{\circ} \mathrm{C}$.

Shao et al. (2012) demonstrated that a combination of chitosan coating plus hot water was more effective in delaying the ripening process and preserving quality than individual application of chitosan coating or hot water.

\section{Compactness}

Data in Table (3) showed that compactness (score) of artichoke heads was significantly decreased with the prolongation of storage period. These results were in agreement with Atala (2006). The decreases in compactness of heads during 
Table 3. Effect of hot water treatment and chitosan coating alone or in a combination between them on compactness (score) of artichoke heads during cold storage.

\begin{tabular}{|c|c|c|c|c|c|c|}
\hline \multirow{3}{*}{ Treatments } & \multicolumn{5}{|c|}{ Storage period(days) } & \multirow{3}{*}{ Mean } \\
\hline & \multicolumn{5}{|c|}{2017 season } & \\
\hline & start & 7days & 14days & 21days & 28days & \\
\hline Chitosan 1\% & $5.00 \mathrm{~A}$ & $5.00 \mathrm{~A}$ & $5.00 \mathrm{~A}$ & $4.33 \mathrm{~A}-\mathrm{C}$ & 3.67 C-E & $4.60 \mathrm{AB}$ \\
\hline Chitosan $1.5 \%$ & $5.00 \mathrm{~A}$ & $5.00 \mathrm{~A}$ & $5.00 \mathrm{~A}$ & $4.00 \mathrm{~B}-\mathrm{D}$ & $3.33 \mathrm{D}-\mathrm{F}$ & 4.47 BC \\
\hline Hot water $44^{\circ} \mathrm{C}$ & $5.00 \mathrm{~A}$ & $5.00 \mathrm{~A}$ & $4.67 \mathrm{AB}$ & 3.67 C-E & $3.00 \mathrm{E}-\mathrm{G}$ & 4.27 CD \\
\hline Hot water $44^{\circ} \mathrm{C}+$ Chitosan $1 \%$ & $5.00 \mathrm{~A}$ & $5.00 \mathrm{~A}$ & $5.00 \mathrm{~A}$ & $5.00 \mathrm{~A}$ & $4.33 \mathrm{~A}-\mathrm{C}$ & 4.87 A \\
\hline Hot water $44^{\circ} \mathrm{C}+$ Chitosan $1.5 \%$ & $5.00 \mathrm{~A}$ & $5.00 \mathrm{~A}$ & $5.00 \mathrm{~A}$ & $4.67 \mathrm{AB}$ & 4.00 B-D & 4.73 AB \\
\hline Hot water $48^{\circ} \mathrm{C}$ & $5.00 \mathrm{~A}$ & $5.00 \mathrm{~A}$ & $3.33 \mathrm{D}-\mathrm{F}$ & $3.00 \mathrm{E}-\mathrm{G}$ & $2.00 \mathrm{HI}$ & $3.67 \mathrm{~F}$ \\
\hline Hot water $48^{\circ} \mathrm{C}+$ Chitosan $1 \%$ & $5.00 \mathrm{~A}$ & $5.00 \mathrm{~A}$ & $4.00 \mathrm{~B}-\mathrm{D}$ & $3.33 \mathrm{D}-\mathrm{F}$ & $2.67 \mathrm{~F}-\mathrm{H}$ & 4.00 DE \\
\hline Hot water $48^{\circ} \mathrm{C}+$ Chitosan $1.5 \%$ & $5.00 \mathrm{~A}$ & $5.00 \mathrm{~A}$ & 3.67 C-E & $2.67 \mathrm{~F}-\mathrm{H}$ & $2.33 \mathrm{GH}$ & 3.73 EF \\
\hline Control & $5.00 \mathrm{~A}$ & $4.33 \mathrm{~A}-\mathrm{C}$ & $3.00 \mathrm{E}-\mathrm{G}$ & $2.00 \mathrm{HI}$ & $1.33 \mathrm{I}$ & $3.13 \mathrm{G}$ \\
\hline Mean & $5.00 \mathrm{~A}$ & $4.93 \mathrm{~A}$ & $4.30 \mathrm{~B}$ & $3.63 \mathrm{C}$ & $2.96 \mathrm{D}$ & \\
\hline \multicolumn{7}{|c|}{2018 season } \\
\hline Chitosan 1\% & $5.00 \mathrm{~A}$ & $5.00 \mathrm{~A}$ & $5.00 \mathrm{~A}$ & $4.67 \mathrm{AB}$ & $4.00 \mathrm{~B}-\mathrm{D}$ & $4.73 \mathrm{AB}$ \\
\hline Chitosan $1.5 \%$ & $5.00 \mathrm{~A}$ & $5.00 \mathrm{~A}$ & $5.00 \mathrm{~A}$ & $4.33 \mathrm{~A}-\mathrm{C}$ & $3.33 \mathrm{D}-\mathrm{F}$ & 4.53 BC \\
\hline Hot water $44^{\circ} \mathrm{C}$ & $5.00 \mathrm{~A}$ & $5.00 \mathrm{~A}$ & $5.00 \mathrm{~A}$ & 4.00 B-D & $3.00 \mathrm{E}-\mathrm{G}$ & $4.40 \mathrm{C}$ \\
\hline Hot water $44^{\circ} \mathrm{C}+$ Chitosan $1 \%$ & $5.00 \mathrm{~A}$ & $5.00 \mathrm{~A}$ & $5.00 \mathrm{~A}$ & $5.00 \mathrm{~A}$ & $4.33 \mathrm{~A}-\mathrm{C}$ & $4.87 \mathrm{~A}$ \\
\hline Hot water $44^{\circ} \mathrm{C}+$ Chitosan $1.5 \%$ & $5.00 \mathrm{~A}$ & $5.00 \mathrm{~A}$ & $5.00 \mathrm{~A}$ & $4.67 \mathrm{AB}$ & 4.00 B-D & $4.73 \mathrm{AB}$ \\
\hline Hot water $48^{\circ} \mathrm{C}$ & $5.00 \mathrm{~A}$ & $5.00 \mathrm{~A}$ & 3.67 C-E & $3.33 \mathrm{D}-\mathrm{F}$ & $2.00 \mathrm{HI}$ & $3.80 \mathrm{D}$ \\
\hline Hot water $48^{\circ} \mathrm{C}+$ Chitosan $1 \%$ & $5.00 \mathrm{~A}$ & $5.00 \mathrm{~A}$ & 4.00 B-D & 3.67 C-E & $2.67 \mathrm{~F}-\mathrm{H}$ & 4.07 D \\
\hline Hot water $48^{\circ} \mathrm{C}+$ Chitosan $1.5 \%$ & $5.00 \mathrm{~A}$ & $5.00 \mathrm{~A}$ & 3.67 C-E & $3.00 \mathrm{E}-\mathrm{G}$ & 2.33 G-I & $3.80 \mathrm{D}$ \\
\hline Control & $5.00 \mathrm{~A}$ & $4.67 \mathrm{AB}$ & $3.00 \mathrm{E}-\mathrm{G}$ & 2.33 G-I & $1.67 \mathrm{I}$ & $3.33 \mathrm{E}$ \\
\hline Mean & $5.00 \mathrm{~A}$ & $4.96 \mathrm{~A}$ & 4.37 B & $3.89 \mathrm{C}$ & 3.04 D & \\
\hline
\end{tabular}

Means in the same column having the same letter are not significantly different at 0.05 level by Duncan's multiple rang test.

Regarding the effect of postharvest treatments, data revealed that there were significant differences between postharvest treatments and untreated control. The highest score of head compactness were obtained by artichoke heads dipped in hot water at 44 ${ }^{\circ} \mathrm{C}$ plus chitosan $1 \%$, hot water at $44^{\circ} \mathrm{C}$ plus chitosan $1.5 \%$ and chitosan $1 \%$ alone with no significant differences between them. Hot water at $48^{\circ} \mathrm{C}$ alone or combination with chitosan at $1 \%$ or $1.5 \%$ treatments were less effective in this concern, while the lowest values of head compactness score were recorded from untreated control. These results were achieved in the two seasons and were in agreement with those obtained by Atala (2006) for hot water.

In general, the interaction between postharvest treatments and storage periods was significant in the two seasons. After 28 days of storage, artichoke heads dipped in hot water at $44^{\circ} \mathrm{C}+$ chitosan $1 \%$, hot water at $44^{\circ} \mathrm{C}+$ chitosan $1.5 \%$ and chitosan $1 \%$ alone resulted in higher compactness score with no significant differences between them, while untreated control gave the lowest ones in the same period in the two seasons.
Color ( $\mathrm{L}$ value)

Changes in lightness ( $\mathrm{L}$ value) were observed during storage compared to initial value. Lightness of artichoke heads was affected by storage time. A decrement in $\mathrm{L}$ value was detected by prolonging the storage period (Table 4), resulted darker color. These results were achieved in the two seasons and in agreement with Mohamedien et al. (2019) on artichoke heads. Ardakani and Mostofi (2019) showed that decreasing in $\mathrm{L}$ value relates to water loss in fruit.

Concerning the effect of postharvest treatments, data showed that there were significant differences among postharvest treatments. Artichoke heads dipped in hot water at $44^{\circ} \mathrm{C}$ plus chitosan $1 \%$, hot water at $44^{\circ} \mathrm{C}$ plus chitosan $1.5 \%$ and chitosan at $1 \%$ alone being the most effective treatment in maintaining the $\mathrm{L}$ values, resulted lighter color with no significant differences between them in the two seasons, while untreated control or hot water at $48^{\circ} \mathrm{C}$ give the lowest one of $\mathrm{L}$ values during storage with no significant differences between them in the two seasons, resulted in darker color. The other treatments were less effective in this concern. These results were agreement with Ardakani and Mostofi (2019) chitosan coated lightened slightly as evidenced by increasing values of $L$ value. 
Table 4. Effect of hot water treatment and chitosan coating alone or in a combination between them on color (L. value) of artichoke heads during cold storage.

\begin{tabular}{|c|c|c|c|c|c|c|}
\hline \multirow{3}{*}{ Treatments } & \multicolumn{5}{|c|}{ Storage period(days) } & \multirow{3}{*}{ Mean } \\
\hline & \multicolumn{5}{|c|}{2017 season } & \\
\hline & start & 7days & 14days & 21days & 28days & \\
\hline Chitosan 1\% & $44.67 \mathrm{~A}$ & $43.73 \mathrm{~A}-\mathrm{D}$ & 42.99 A-E & $40.98 \mathrm{D}-\mathrm{H}$ & 39.07 F-J & 42.29 A-C \\
\hline Chitosan $1.5 \%$ & $44.67 \mathrm{~A}$ & 43.34 A-D & $42.78 \mathrm{~A}-\mathrm{E}$ & $40.05 \mathrm{E}-\mathrm{I}$ & $38.07 \mathrm{H}-\mathrm{J}$ & 41.78 B-D \\
\hline Hot water $44^{\circ} \mathrm{C}$ & $44.67 \mathrm{~A}$ & 43.23 A-D & $42.37 \mathrm{~A}-\mathrm{E}$ & 39.97 E-I & $37.65 \mathrm{IJ}$ & 41.58 B-D \\
\hline Hot water $44^{\circ} \mathrm{C}+$ Chitosan $1 \%$ & $44.67 \mathrm{~A}$ & $44.50 \mathrm{AB}$ & 43.68 A-D & 42.79 A-E & $41.70 \mathrm{~A}-\mathrm{G}$ & 43.47 A \\
\hline Hot water $44^{\circ} \mathrm{C}+$ Chitosan $1.5 \%$ & $44.67 \mathrm{~A}$ & $44.17 \mathrm{~A}-\mathrm{C}$ & $43.35 \mathrm{~A}-\mathrm{D}$ & $41.47 \mathrm{~B}-\mathrm{G}$ & $40.07 \mathrm{E}-\mathrm{I}$ & $42.75 \mathrm{AB}$ \\
\hline Hot water $48^{\circ} \mathrm{C}$ & $44.67 \mathrm{~A}$ & $41.97 \mathrm{~A}-\mathrm{G}$ & 40.16 E-I & $37.89 \mathrm{IJ}$ & $32.92 \mathrm{~K}$ & $39.52 \mathrm{EF}$ \\
\hline Hot water $48^{\circ} \mathrm{C}+$ Chitosan $1 \%$ & $44.67 \mathrm{~A}$ & $42.42 \mathrm{~A}-\mathrm{E}$ & $41.93 \mathrm{~A}-\mathrm{G}$ & $39.21 \mathrm{~F}-J$ & $37.18 \mathrm{IJ}$ & 41.08 CD \\
\hline Hot water $48^{\circ} \mathrm{C}+$ Chitosan $1.5 \%$ & $44.67 \mathrm{~A}$ & $42.10 \mathrm{~A}-\mathrm{F}$ & $41.34 \mathrm{C}-\mathrm{G}$ & 39.07 F-J & $36.86 \mathrm{~J}$ & 40.81 DE \\
\hline Control & $44.67 \mathrm{~A}$ & $41.57 \mathrm{~B}-\mathrm{G}$ & 39.03 G-J & $36.53 \mathrm{~J}$ & $32.13 \mathrm{~K}$ & $38.79 \mathrm{~F}$ \\
\hline Mean & 44.67 A & 43.00 B & $41.96 \mathrm{C}$ & 39.77 D & $37.30 \mathrm{E}$ & \\
\hline \multicolumn{7}{|c|}{2018 season } \\
\hline Chitosan 1\% & $42.30 \mathrm{~A}$ & $41.10 \mathrm{~A}-\mathrm{C}$ & $40.95 \mathrm{~A}-\mathrm{C}$ & $39.49 \mathrm{~A}-\mathrm{G}$ & $38.79 \mathrm{~B}-\mathrm{I}$ & $40.52 \mathrm{~A}-\mathrm{C}$ \\
\hline Chitosan $1.5 \%$ & $42.30 \mathrm{~A}$ & $40.86 \mathrm{~A}-\mathrm{D}$ & $40.47 \mathrm{~A}-\mathrm{E}$ & 39.34 A-H & $37.16 \mathrm{~F}-\mathrm{K}$ & 40.03 B-D \\
\hline Hot water $44^{\circ} \mathrm{C}$ & $42.30 \mathrm{~A}$ & $40.72 \mathrm{~A}-\mathrm{E}$ & 40.29 A-E & 38.75 B-I & $36.27 \mathrm{I}-\mathrm{L}$ & 39.67 CD \\
\hline Hot water $4^{\circ} \mathrm{C}+$ Chitosan $1 \%$ & $42.30 \mathrm{~A}$ & $41.95 \mathrm{~A}$ & $41.62 \mathrm{AB}$ & $41.23 \mathrm{~A}-\mathrm{C}$ & $40.48 \mathrm{~A}-\mathrm{E}$ & $41.52 \mathrm{~A}$ \\
\hline Hot water $44^{\circ} \mathrm{C}+$ Chitosan $1.5 \%$ & $42.30 \mathrm{~A}$ & $41.73 \mathrm{AB}$ & $41.09 \mathrm{~A}-\mathrm{C}$ & $40.46 \mathrm{~A}-\mathrm{E}$ & $39.81 \mathrm{~A}-\mathrm{F}$ & 41.08 AB \\
\hline Hot water $48^{\circ} \mathrm{C}$ & $42.30 \mathrm{~A}$ & 38.75 B-I & 36.47 G-L & $35.57 \mathrm{~J}-\mathrm{M}$ & $33.08 \mathrm{MN}$ & $37.23 \mathrm{~F}$ \\
\hline Hot water $48^{\circ} \mathrm{C}+$ Chitosan $1 \%$ & $42.30 \mathrm{~A}$ & $40.33 \mathrm{~A}-\mathrm{E}$ & $39.30 \mathrm{~A}-\mathrm{I}$ & 37.89 D-K & $34.00 \mathrm{~L}-\mathrm{N}$ & 38.76 DE \\
\hline Hot water $48^{\circ} \mathrm{C}+$ Chitosan $1.5 \%$ & $42.30 \mathrm{~A}$ & $39.46 \mathrm{~A}-\mathrm{G}$ & $37.75 \mathrm{E}-\mathrm{K}$ & $36.89 \mathrm{~F}-\mathrm{L}$ & $33.13 \mathrm{MN}$ & $37.91 \mathbf{E}$ \\
\hline Control & $42.30 \mathrm{~A}$ & $38.52 \mathrm{C}-\mathrm{J}$ & $36.37 \mathrm{H}-\mathrm{L}$ & $35.37 \mathrm{~K}-\mathrm{M}$ & $32.05 \mathrm{~N}$ & $36.92 \mathrm{~F}$ \\
\hline Mean & $42.30 \mathrm{~A}$ & 40.38 B & 39.37 B & $38.33 \mathrm{C}$ & $36.08 \mathrm{D}$ & \\
\hline
\end{tabular}

Means in the same column having the same letter are not significantly different at 0.05 level by Duncan's multiple rang tes

\section{Color (Hue angle values)}

Changes in hue angles of artichoke heads are good indicator of senescence. As shown in Table (5) showed that the hue angle values of artichoke heads gradually decreased as the storage period extended, indicating that artichoke heads turned to slight yellow as the storage period prolonged. These results were true in the two seasons and in agreement with (Atala, 2006; Mohamedien et al., 2019).

Concerning the effect of postharvest treatments, data showed that all treatments had significantly higher hue angle values as compared with untreated control. Heads dipped in hot water at $44^{\circ} \mathrm{C}+$ chitosan $1 \%$, hot water at $44^{\circ} \mathrm{C}+$ chitosan $1.5 \%$ and chitosan at $1 \%$ alone were the most effective treatments in reducing the loss of hue angle values indicated that heads retained more green color (higher value of hue angle) with no significant differences between them in the two seasons during storage. On the other hand, untreated control gave the lower value of hue angle as an important degreening or intense yellowing. These results were true in the two seasons and in agreement with Ahmed et al. (2016) for chitosan; Shehata et al. (2013) for hot water; Chailoo and Asghari (2011) for combined with hot water plus chitosan.

The reduction of color development in samples treated with hot water plus chitosan could be attributed to the low rate of respiration rate and reduced ethylene production (Djioua et al., 2010) resulted in lower activity of chlorophylls and chlorophyll degradation, so maintained green color of fruits (Jianglian and Shaoying, 2013).

\section{Dry matter percentage}

Data in Table (6) showed that dry matter of heads was significantly decreased with the prolongation of storage period. Similar results were obtained by Atala (2006). Respiration is a chemical process necessary for all living tissue whereby starch and sugars (dry matter) are oxidized to carbon dioxide and water vapor with the liberation of heat (Picha, 1987). 
Table 5. Effect of hot water treatment and chitosan coating alone or in a combination between them on color (hue angle $\mathrm{h}^{\circ}$ ) of artichoke heads during cold storage.

\begin{tabular}{|c|c|c|c|c|c|c|}
\hline \multirow{3}{*}{ Treatments } & \multicolumn{5}{|c|}{ Storage period(days) } & \multirow{3}{*}{ Mean } \\
\hline & \multicolumn{5}{|c|}{2017 season } & \\
\hline & start & 7days & 14 days & 21days & 28days & \\
\hline Chitosan 1\% & $93.56 \mathrm{~A}$ & $92.87 \mathrm{~A}-\mathrm{C}$ & $91.21 \mathrm{~A}-\mathrm{G}$ & 89.88 A-I & $86.96 \mathrm{H}-\mathrm{M}$ & $90.90 \mathrm{~A}-\mathrm{C}$ \\
\hline Chitosan $1.5 \%$ & $93.56 \mathrm{~A}$ & $92.05 \mathrm{~A}-\mathrm{F}$ & $90.18 \mathrm{~A}-\mathrm{H}$ & 89.06 C-J & $86.02 \mathrm{I}-\mathrm{N}$ & 90.18 B-D \\
\hline Hot water $44^{\circ} \mathrm{C}$ & $93.56 \mathrm{~A}$ & $92.11 \mathrm{~A}-\mathrm{F}$ & $90.05 \mathrm{~A}-\mathrm{H}$ & $88.60 \mathrm{E}-\mathrm{K}$ & $84.99 \mathrm{~K}-\mathrm{N}$ & 89.86 B-D \\
\hline Hot water $44^{\circ} \mathrm{C}+$ Chitosan $1 \%$ & $93.56 \mathrm{~A}$ & $93.30 \mathrm{AB}$ & $92.61 \mathrm{~A}-\mathrm{D}$ & $91.13 \mathrm{~A}-\mathrm{G}$ & $89.51 \mathrm{~B}-\mathrm{J}$ & $92.02 \mathrm{~A}$ \\
\hline $\begin{array}{l}\text { Hot water } 44^{\circ} \mathrm{C}+\text { Chitosan } \\
1.5 \%\end{array}$ & $93.56 \mathrm{~A}$ & $93.09 \mathrm{AB}$ & $92.35 \mathrm{~A}-\mathrm{E}$ & $90.46 \mathrm{~A}-\mathrm{H}$ & $88.25 \mathrm{~F}-\mathrm{L}$ & 91.54 AB \\
\hline Hot water $48^{\circ} \mathrm{C}$ & $93.56 \mathrm{~A}$ & $91.54 \mathrm{~A}-\mathrm{G}$ & $88.60 \mathrm{D}-\mathrm{K}$ & $85.72 \mathrm{~J}-\mathrm{N}$ & $83.50 \mathrm{M}-\mathrm{O}$ & 88.59 D \\
\hline Hot water $48^{\circ} \mathrm{C}+$ Chitosan $1 \%$ & $93.56 \mathrm{~A}$ & $92.00 \mathrm{~A}-\mathrm{F}$ & 89.99 A-I & $87.89 \mathrm{G}-\mathrm{L}$ & $84.72 \mathrm{~K}-\mathrm{N}$ & 89.63 CD \\
\hline Hot water $48^{\circ} \mathrm{C}+$ Chitosan $1.5 \%$ & $93.56 \mathrm{~A}$ & $91.77 \mathrm{~A}-\mathrm{G}$ & $88.50 \mathrm{E}-\mathrm{K}$ & $86.91 \mathrm{H}-\mathrm{N}$ & $84.25 \mathrm{~L}-\mathrm{O}$ & 89.00 D \\
\hline Control & $93.56 \mathrm{~A}$ & $85.04 \mathrm{~K}-\mathrm{N}$ & $82.94 \mathrm{NO}$ & $80.30 \mathrm{OP}$ & $78.43 \mathrm{P}$ & 84.05 E \\
\hline Mean & $93.56 \mathrm{~A}$ & $91.53 \mathrm{~B}$ & $89.60 \mathrm{C}$ & 87.77 D & 85.18 E & \\
\hline \multicolumn{7}{|c|}{2018 season } \\
\hline Chitosan 1\% & $91.59 \mathrm{~A}$ & $90.91 \mathrm{~A}-\mathrm{C}$ & $89.38 \mathrm{~A}-\mathrm{H}$ & $87.18 \mathrm{~F}-\mathrm{M}$ & $84.92 \mathrm{~L}-\mathrm{R}$ & $88.80 \mathrm{~A}-\mathrm{C}$ \\
\hline Chitosan $1.5 \%$ & $91.59 \mathrm{~A}$ & $90.51 \mathrm{~A}-\mathrm{D}$ & 88.76 A-J & $86.68 \mathrm{H}-\mathrm{N}$ & $83.98 \mathrm{~N}-\mathrm{R}$ & 88.30 B-D \\
\hline Hot water $44^{\circ} \mathrm{C}$ & $91.59 \mathrm{~A}$ & $90.04 \mathrm{~A}-\mathrm{F}$ & 88.43 B-J & $86.25 \mathrm{I}-\mathrm{O}$ & $83.29 \mathrm{O}-\mathrm{R}$ & 87.92 C-E \\
\hline Hot water $44^{\circ} \mathrm{C}+$ Chitosan $1 \%$ & $91.59 \mathrm{~A}$ & $91.48 \mathrm{AB}$ & 90.75 A-D & $89.21 \mathrm{~A}-\mathrm{I}$ & 87.24 E-M & $90.05 \mathrm{~A}$ \\
\hline Hot water $44^{\circ} \mathrm{C}+$ Chitosan $1.5 \%$ & $91.59 \mathrm{~A}$ & $91.05 \mathrm{~A}-\mathrm{C}$ & $90.30 \mathrm{~A}-\mathrm{E}$ & $88.06 \mathrm{C}-\mathrm{K}$ & $86.66 \mathrm{H}-\mathrm{N}$ & 89.53 AB \\
\hline Hot water $48^{\circ} \mathrm{C}$ & $91.59 \mathrm{~A}$ & 89.32 A-I & $86.89 \mathrm{G}-\mathrm{N}$ & 84.30 M-R & $81.85 \mathrm{RS}$ & $86.79 \mathrm{E}$ \\
\hline Hot water $48^{\circ} \mathrm{C}+$ Chitosan $1 \%$ & $91.59 \mathrm{~A}$ & $89.90 \mathrm{~A}-\mathrm{G}$ & 87.67 D-L & $85.70 \mathrm{~J}-\mathrm{P}$ & 82.83 P-R & 87.54 C-E \\
\hline Hot water $48^{\circ} \mathrm{C}+$ Chitosan $1.5 \%$ & $91.59 \mathrm{~A}$ & $89.74 \mathrm{~A}-\mathrm{H}$ & 87.08 F-M & 85.20 K-Q & 82.29 Q-S & 87.18 DE \\
\hline Control & $91.59 \mathrm{~A}$ & $85.06 \mathrm{~K}-\mathrm{Q}$ & 82.18 Q-S & $79.70 \mathrm{ST}$ & $77.15 \mathrm{~T}$ & 83.14 F \\
\hline Mean & $91.59 \mathrm{~A}$ & 89.78 B & $87.94 \mathrm{C}$ & 85.81 D & 83.36 E & \\
\hline
\end{tabular}

Means in the same column having the same letter are not significantly different at 0.05 level by Duncan's multiple rang test.

Table 6. Effect of hot water treatment and chitosan coating alone or in a combination between them on dry mater percentage of artichoke heads during cold storage.

\begin{tabular}{|c|c|c|c|c|c|c|}
\hline \multirow{3}{*}{ Treatments } & \multicolumn{5}{|c|}{ Storage period(days) } & \multirow{3}{*}{ Mean } \\
\hline & \multicolumn{5}{|c|}{2017 season } & \\
\hline & start & 7days & 14days & 21days & 28days & \\
\hline Chitosan 1\% & $21.94 \mathrm{~A}$ & $21.48 \mathrm{AB}$ & $20.33 \mathrm{C}-\mathrm{E}$ & $18.69 \mathrm{G}-\mathrm{J}$ & $16.08 \mathrm{PQ}$ & $19.70 \mathrm{~B}$ \\
\hline Chitosan $1.5 \%$ & $21.94 \mathrm{~A}$ & $20.47 \mathrm{C}-\mathrm{E}$ & 19.87 D-F & $18.07 \mathrm{~J}-\mathrm{L}$ & $15.61 \mathrm{Q}$ & $19.19 \mathrm{C}$ \\
\hline Hot water $44^{\circ} \mathrm{C}$ & $21.94 \mathrm{~A}$ & $19.78 \mathrm{EF}$ & $19.30 \mathrm{~F}-\mathrm{H}$ & $17.58 \mathrm{~K}-\mathrm{N}$ & $14.55 \mathrm{RS}$ & 18.63 D \\
\hline Hot water $44^{\circ} \mathrm{C}+$ Chitosan $1 \%$ & $21.94 \mathrm{~A}$ & $21.71 \mathrm{AB}$ & $21.27 \mathrm{~A}-\mathrm{C}$ & 20.42 C-E & $18.23 \mathrm{I}-\mathrm{L}$ & $20.71 \mathrm{~A}$ \\
\hline Hot water $44^{\circ} \mathrm{C}+$ Chitosan $1.5 \%$ & $21.94 \mathrm{~A}$ & $21.55 \mathrm{AB}$ & 20.83 B-D & 19.20 F-I & $16.89 \mathrm{M}-\mathrm{P}$ & 20.08 B \\
\hline Hot water $48^{\circ} \mathrm{C}$ & $21.94 \mathrm{~A}$ & 19.16 F-I & $18.05 \mathrm{~J}-\mathrm{L}$ & $16.37 \mathrm{O}-\mathrm{Q}$ & $12.69 \mathrm{UV}$ & $17.64 \mathrm{~F}$ \\
\hline Hot water $48^{\circ} \mathrm{C}+$ Chitosan $1 \%$ & $21.94 \mathrm{~A}$ & $19.62 \mathrm{E}-\mathrm{G}$ & $19.18 \mathrm{~F}-\mathrm{I}$ & 17.34 L-O & $13.97 \mathrm{ST}$ & 18.41 DE \\
\hline Hot water $48^{\circ} \mathrm{C}+$ Chitosan $1.5 \%$ & $21.94 \mathrm{~A}$ & $19.28 \mathrm{~F}-\mathrm{H}$ & $18.41 \mathrm{H}-\mathrm{K}$ & $16.80 \mathrm{~N}-\mathrm{P}$ & $13.45 \mathrm{TU}$ & $17.98 \mathrm{EF}$ \\
\hline Control & $21.94 \mathrm{~A}$ & $18.12 \mathrm{~J}-\mathrm{L}$ & $17.80 \mathrm{~J}-\mathrm{M}$ & $15.49 \mathrm{QR}$ & $11.74 \mathrm{~V}$ & $17.02 \mathrm{G}$ \\
\hline Mean & $21.94 \mathrm{~A}$ & 20.13 B & 19.45 C & $17.77 \mathrm{D}$ & $14.80 \mathrm{E}$ & \\
\hline \multicolumn{7}{|c|}{2018 season } \\
\hline Chitosan $1 \%$ & $20.43 \mathrm{~A}$ & $19.99 \mathrm{~A}-\mathrm{C}$ & $18.86 \mathrm{C}-\mathrm{E}$ & $17.20 \mathrm{~F}-\mathrm{I}$ & $14.57 \mathrm{M}-\mathrm{O}$ & 18.21 BC \\
\hline Chitosan $1.5 \%$ & $20.43 \mathrm{~A}$ & 18.98 B-E & $18.40 \mathrm{D}-\mathrm{F}$ & $16.58 \mathrm{H}-\mathrm{K}$ & $14.10 \mathrm{~N}-\mathrm{P}$ & $17.70 \mathrm{CD}$ \\
\hline Hot water $44^{\circ} \mathrm{C}$ & $20.43 \mathrm{~A}$ & $18.29 \mathrm{D}-\mathrm{G}$ & $17.83 \mathrm{E}-\mathrm{H}$ & $16.09 \mathrm{I}-\mathrm{L}$ & $13.05 \mathrm{PQ}$ & 17.14 DE \\
\hline Hot water $44^{\circ} \mathrm{C}+$ Chitosan $1 \%$ & $20.43 \mathrm{~A}$ & $20.22 \mathrm{AB}$ & $19.80 \mathrm{~A}-\mathrm{C}$ & 18.93 B-E & $16.72 \mathrm{H}-\mathrm{J}$ & $19.22 \mathrm{~A}$ \\
\hline Hot water $44^{\circ} \mathrm{C}+$ Chitosan $1.5 \%$ & $20.43 \mathrm{~A}$ & $20.06 \mathrm{~A}-\mathrm{C}$ & 19.36 A-D & $17.72 \mathrm{E}-\mathrm{H}$ & $15.38 \mathrm{~J}-\mathrm{N}$ & $18.59 \mathrm{~B}$ \\
\hline Hot water $48^{\circ} \mathrm{C}$ & $20.43 \mathrm{~A}$ & 17.67 E-H & $16.58 \mathrm{H}-\mathrm{K}$ & $14.88 \mathrm{~L}-\mathrm{O}$ & $11.18 \mathrm{RS}$ & $16.15 \mathrm{G}$ \\
\hline Hot water $48^{\circ} \mathrm{C}+$ Chitosan $1 \%$ & $20.43 \mathrm{~A}$ & $18.13 \mathrm{D}-\mathrm{G}$ & $17.71 \mathrm{E}-\mathrm{H}$ & $15.85 \mathrm{I}-\mathrm{M}$ & $12.47 \mathrm{QR}$ & $16.92 \mathrm{EF}$ \\
\hline Hot water $48^{\circ} \mathrm{C}+$ Chitosan $1.5 \%$ & $20.43 \mathrm{~A}$ & $17.79 \mathrm{E}-\mathrm{H}$ & 16.94 G-I & $15.31 \mathrm{~K}-\mathrm{N}$ & $11.94 \mathrm{QR}$ & 16.48 FG \\
\hline Control & $20.43 \mathrm{~A}$ & $16.63 \mathrm{H}-\mathrm{K}$ & $16.27 \mathrm{I}-\mathrm{K}$ & $13.93 \mathrm{OP}$ & $10.16 \mathrm{~S}$ & $15.48 \mathrm{H}$ \\
\hline Mean & $20.43 \mathrm{~A}$ & 18.64 B & 17.97 C & $16.28 \mathrm{D}$ & $13.29 \mathrm{E}$ & \\
\hline
\end{tabular}

Means in the same column having the same letter are not significantly different at 0.05 level by Duncan's 
Regarding the effect of postharvest treatments, data revealed that all postharvest treatments were effective in maintaining dry matter percentage during storage as compared with untreated control. Moreover, artichoke heads dipped in hot water at $44^{\circ} \mathrm{C}$ plus chitosan $1 \%$ was the most effective treatment in maintaining dry matter followed by hot water at $44^{\circ} \mathrm{C}$ plus chitosan $1.5 \%$ and chitosan $1 \%$ in the two seasons. The lowest values resulted in untreated control. These results were achieved in the two seasons and were in agreement with those obtained by Atala (2006) and El-Sayed et al. (2013) for hot water.

In general, the interaction between postharvest treatments and storage periods was significant in the two seasons. After 28 days of storage, artichoke heads dipped in hot water at $44^{\circ} \mathrm{C}$ plus chitosan $1 \%$ and hot water at $44^{\circ} \mathrm{C}$ plus chitosan $1.5 \%$ resulted in higher dry matter percentage with no significant differences between them in the second season, while untreated control gave the lowest ones in the same period in the two seasons.

\section{Ascorbic acid content}

Data in Table (7) showed that ascorbic acid content of heads was significantly decreased with the prolongation of storage period. Similar results were obtained by (Mohamedien et al., 2019).

The reduction in ascorbic acid contents during storage may be due to the higher rate of sugar loss through respiration than the water loss through transpiration (Wills et al., 1998).

Table 7. Effect of hot water treatment and chitosan coating alone or in a combination between them on ascorbic acid content (mg/100gm f.w) of artichoke heads during cold storage.

\begin{tabular}{|c|c|c|c|c|c|c|}
\hline \multirow{3}{*}{ Treatments } & \multicolumn{5}{|c|}{ Storage period(days) } & \multirow{3}{*}{ Mean } \\
\hline & \multicolumn{5}{|c|}{2017 season } & \\
\hline & start & 7days & 14days & 21days & 28days & \\
\hline Chitosan 1\% & $81.33 \mathrm{~A}$ & $80.80 \mathrm{AB}$ & $79.13 \mathrm{~A}-\mathrm{F}$ & $76.33 \mathrm{~A}-\mathrm{H}$ & $73.73 \mathrm{E}-\mathrm{I}$ & $78.27 \mathrm{AB}$ \\
\hline Chitosan 1.5\% & $81.33 \mathrm{~A}$ & $80.43 \mathrm{AB}$ & $78.93 \mathrm{~A}-\mathrm{F}$ & 75.80 A-I & 72.27 G-J & $77.75 \mathrm{~A}-\mathrm{C}$ \\
\hline Hot water $44^{\circ} \mathrm{C}$ & $81.33 \mathrm{~A}$ & $80.40 \mathrm{~A}-\mathrm{C}$ & $78.20 \mathrm{~A}-\mathrm{G}$ & 75.20 A-I & $71.00 \mathrm{H}-\mathrm{K}$ & 77.23 A-C \\
\hline Hot water $44^{\circ} \mathrm{C}+$ Chitosan $1 \%$ & $81.33 \mathrm{~A}$ & $81.07 \mathrm{AB}$ & $80.00 \mathrm{~A}-\mathrm{D}$ & $79.00 \mathrm{~A}-\mathrm{F}$ & $77.33 \mathrm{~A}-\mathrm{G}$ & $79.75 \mathrm{~A}$ \\
\hline Hot water $44^{\circ} \mathrm{C}+$ Chitosan $1.5 \%$ & $81.33 \mathrm{~A}$ & $80.93 \mathrm{AB}$ & $79.33 \mathrm{~A}-\mathrm{E}$ & $77.13 \mathrm{~A}-\mathrm{H}$ & $75.00 \mathrm{~B}-\mathrm{I}$ & $78.75 \mathrm{AB}$ \\
\hline Hot water $48^{\circ} \mathrm{C}$ & $81.33 \mathrm{~A}$ & 79.80 A-E & $76.47 \mathrm{~A}-\mathrm{H}$ & 72.33 G-J & $66.67 \mathrm{JK}$ & 75.32 CD \\
\hline Hot water $48^{\circ} \mathrm{C}+$ Chitosan $1 \%$ & $81.33 \mathrm{~A}$ & $80.20 \mathrm{~A}-\mathrm{D}$ & $77.80 \mathrm{~A}-\mathrm{G}$ & 74.20 C-I & $70.93 \mathrm{H}-\mathrm{K}$ & 76.89 B-D \\
\hline Hot water $48^{\circ} \mathrm{C}+$ Chitosan $1.5 \%$ & $81.33 \mathrm{~A}$ & $80.13 \mathrm{~A}-\mathrm{D}$ & $77.40 \mathrm{~A}-\mathrm{G}$ & $73.00 \mathrm{~F}-\mathrm{I}$ & $69.73 \mathrm{I}-\mathrm{K}$ & 76.32 B-D \\
\hline Control & $81.33 \mathrm{~A}$ & $79.60 \mathrm{~A}-\mathrm{E}$ & $74.00 \mathrm{D}-\mathrm{I}$ & $70.07 \mathrm{I}-\mathrm{K}$ & $65.87 \mathrm{~K}$ & 74.17 D \\
\hline Mean & $81.33 \mathrm{~A}$ & 80.37 A & 77.92 B & $74.79 \mathrm{C}$ & 71.39 D & \\
\hline \multicolumn{7}{|c|}{2018 season } \\
\hline Chitosan 1\% & $78.93 \mathrm{~A}$ & $77.87 \mathrm{~A}-\mathrm{C}$ & $76.73 \mathrm{~A}-\mathrm{E}$ & $73.60 \mathrm{~A}-\mathrm{H}$ & $70.47 \mathrm{D}-\mathrm{I}$ & $75.52 \mathrm{~A}-\mathrm{C}$ \\
\hline Chitosan $1.5 \%$ & $78.93 \mathrm{~A}$ & $77.67 \mathrm{~A}-\mathrm{C}$ & $75.73 \mathrm{~A}-\mathrm{F}$ & 72.87 A-I & $69.13 \mathrm{~F}-\mathrm{J}$ & 74.87 A-D \\
\hline Hot water $44^{\circ} \mathrm{C}$ & $78.93 \mathrm{~A}$ & $77.53 \mathrm{~A}-\mathrm{C}$ & $75.27 \mathrm{~A}-\mathrm{F}$ & $72.20 \mathrm{~B}-\mathrm{I}$ & $68.27 \mathrm{G}-\mathrm{K}$ & 74.44 A-D \\
\hline Hot water $44^{\circ} \mathrm{C}+$ Chitosan $1 \%$ & $78.93 \mathrm{~A}$ & $78.33 \mathrm{AB}$ & $77.80 \mathrm{~A}-\mathrm{C}$ & 76.47 A-E & $74.67 \mathrm{~A}-\mathrm{G}$ & $77.24 \mathrm{~A}$ \\
\hline Hot water $44^{\circ} \mathrm{C}+$ Chitosan $1.5 \%$ & $78.93 \mathrm{~A}$ & $78.00 \mathrm{~A}-\mathrm{C}$ & 77.07 A-D & $75.00 \mathrm{~A}-\mathrm{F}$ & $73.60 \mathrm{~A}-\mathrm{H}$ & $76.52 \mathrm{AB}$ \\
\hline Hot water $48^{\circ} \mathrm{C}$ & $78.93 \mathrm{~A}$ & $76.87 \mathrm{~A}-\mathrm{E}$ & $73.60 \mathrm{~A}-\mathrm{H}$ & 69.33 F-J & $63.47 \mathrm{JK}$ & $72.44 \mathrm{DE}$ \\
\hline Hot water $48^{\circ} \mathrm{C}+$ Chitosan $1 \%$ & $78.93 \mathrm{~A}$ & $77.40 \mathrm{~A}-\mathrm{C}$ & $74.93 \mathrm{~A}-\mathrm{F}$ & 71.47 C-I & $67.20 \mathrm{H}-\mathrm{K}$ & 73.99 B-E \\
\hline Hot water $48^{\circ} \mathrm{C}+$ Chitosan $1.5 \%$ & $78.93 \mathrm{~A}$ & 77.07 A-D & 74.67 A-G & $70.40 \mathrm{E}-\mathrm{I}$ & $66.40 \mathrm{I}-\mathrm{K}$ & 73.49 C-E \\
\hline Control & $78.93 \mathrm{~A}$ & $76.40 \mathrm{~A}-\mathrm{E}$ & $71.47 \mathrm{C}-\mathrm{I}$ & $67.00 \mathrm{H}-\mathrm{K}$ & $62.47 \mathrm{~K}$ & $71.25 \mathrm{E}$ \\
\hline Mean & $78.93 \mathrm{~A}$ & $77.46 \mathrm{~A}$ & $75.25 \mathrm{~B}$ & 72.04 C & 68.41 D & \\
\hline
\end{tabular}

Means in the same column having the same letter are not significantly different at 0.05 level by Duncan's Multiple rang test.

Regarding the effect of postharvest treatments, data showed that chitosan at $1 \%$ or $1.5 \%$ alone and hot water at $44^{\circ} \mathrm{C}$ alone or combination with chitosan at $1 \%$ or $1.5 \%$ were the most effective treatments in maintaining ascorbic acid content with no significant differences between them in the two seasons. The lowest values resulted in other treatments and untreated control. These results were achieved in the two seasons and were in agreement with those obtained by Jongsri et al.(2016) and Suseno et al. (2014) for chitosan and Abdalla (2013) and Sakaldas and Kaynas (2010) for hot water.

Coverings such as chitosan increase the activity of cytochrome oxidase by decreasing the internal $\mathrm{O}_{2}$ in fruits, and this enzyme can decrease significantly the decomposition rate of ascorbic acid (Özden and Bayindirli, 2002). Mahmoud et al. (2017) found that chitosan treatment succeeded to inhibit damage which causing oxidation of ascorbic acid in Navel orange during storage in both seasons. While, the minimum of vitamin $\mathrm{C}$ in control fruits probably due to physiological disorders, decay and weight loss which led to rapid the oxidation reaction in vitamin $\mathrm{C}$, so decreased and showed the lowest level in control fruits.

Hot water treatments were found effective on preventing vitamin $\mathrm{C}$ degradation during storage. Hot 
water prevented vitamin $\mathrm{C}$ degradation caused by low $\mathrm{O}_{2}$ respiration rate (Sakaldas and Kaynas, 2010).

In general, the interaction between postharvest treatments and storage periods was significant in the two seasons. After 28 days of storage, artichoke heads dipped in hot water at $44^{\circ} \mathrm{C}+$ chitosan $1 \%$ and hot water at $44^{\circ} \mathrm{C}+$ chitosan $1.5 \%$ resulted in higher ascorbic acid content with no significant differences between them in the two seasons, while untreated control gave the lowest ones in the same period in the two seasons.

\section{Total soluble solids percentage}

Data in Table (8) showed that total soluble solids of heads were significantly decreased with the prolongation of storage period. Similar results were obtained by Atala (2006) and Shehata et al. (2013). The loss of total soluble solids during the storage period is natural, as sugars are the primary constituent of the soluble solid contents of product, consumed by respiration and used for the metabolic activities of the fruits (Wills et al., 1998).

Table 8. Effect of hot water treatment and chitosan coating alone or in a combination between them on total soluble solids (\%) of artichoke heads during cold storage.

\begin{tabular}{|c|c|c|c|c|c|c|}
\hline \multirow{3}{*}{ Treatments } & \multicolumn{5}{|c|}{ Storage period(days) } & \multirow{3}{*}{ Mean } \\
\hline & \multicolumn{5}{|c|}{2017 season } & \\
\hline & start & 7days & 14days & 21days & 28days & \\
\hline Chitosan $1 \%$ & $15.10 \mathrm{~A}$ & $15.00 \mathrm{AB}$ & 14.77 A-E & $14.43 \mathrm{~A}-\mathrm{G}$ & $13.83 \mathrm{G}-\mathrm{K}$ & $14.63 \mathrm{~A}-\mathrm{C}$ \\
\hline Chitosan $1.5 \%$ & $15.10 \mathrm{~A}$ & $14.93 \mathrm{~A}-\mathrm{C}$ & $14.60 \mathrm{~A}-\mathrm{F}$ & 14.20 D-I & $13.70 \mathrm{H}-\mathrm{L}$ & 14.51 B-D \\
\hline Hot water $44^{\circ} \mathrm{C}$ & $15.10 \mathrm{~A}$ & 14.90 A-D & $14.53 \mathrm{~A}-\mathrm{G}$ & $14.03 \mathrm{~F}-\mathrm{K}$ & $13.57 \mathrm{I}-\mathrm{M}$ & $14.43 \mathrm{~B}-\mathrm{E}$ \\
\hline Hot water $44^{\circ} \mathrm{C}+$ Chitosan $1 \%$ & $15.10 \mathrm{~A}$ & $15.07 \mathrm{AB}$ & $15.03 \mathrm{AB}$ & $14.80 \mathrm{~A}-\mathrm{E}$ & $14.53 \mathrm{~A}-\mathrm{G}$ & $14.91 \mathrm{~A}$ \\
\hline Hot water $44^{\circ} \mathrm{C}+$ Chitosan $1.5 \%$ & $15.10 \mathrm{~A}$ & $15.03 \mathrm{AB}$ & $14.83 \mathrm{~A}-\mathrm{D}$ & $14.50 \mathrm{~A}-\mathrm{G}$ & $14.10 \mathrm{E}-\mathrm{J}$ & $14.71 \mathrm{AB}$ \\
\hline Hot water $48^{\circ} \mathrm{C}$ & $15.10 \mathrm{~A}$ & 14.77 A-E & 14.37 B-H & $13.57 \mathrm{I}-\mathrm{M}$ & $12.87 \mathrm{MN}$ & $14.13 \mathrm{EF}$ \\
\hline Hot water $48^{\circ} \mathrm{C}+$ Chitosan $1 \%$ & $15.10 \mathrm{~A}$ & $14.83 \mathrm{~A}-\mathrm{D}$ & $14.47 \mathrm{~A}-\mathrm{G}$ & $13.83 \mathrm{G}-\mathrm{K}$ & $13.33 \mathrm{~K}-\mathrm{N}$ & $14.31 \mathrm{C}-\mathrm{F}$ \\
\hline Hot water $48^{\circ} \mathrm{C}+$ Chitosan $1.5 \%$ & $15.10 \mathrm{~A}$ & $14.80 \mathrm{~A}-\mathrm{E}$ & $14.40 \mathrm{~A}-\mathrm{H}$ & $13.70 \mathrm{H}-\mathrm{L}$ & $13.00 \mathrm{~L}-\mathrm{N}$ & 14.20 D-F \\
\hline Control & $15.10 \mathrm{~A}$ & 14.73 A-F & 14.27 C-I & $13.43 \mathrm{~J}-\mathrm{M}$ & $12.67 \mathrm{~N}$ & $14.04 \mathrm{~F}$ \\
\hline Mean & $15.10 \mathrm{~A}$ & $14.90 \mathrm{~A}$ & $14.59 \mathrm{~B}$ & $14.06 \mathrm{C}$ & 13.51 D & \\
\hline \multicolumn{7}{|c|}{2018 season } \\
\hline Chitosan $1 \%$ & $14.33 \mathrm{~A}$ & $14.20 \mathrm{~A}-\mathrm{C}$ & $13.93 \mathrm{~A}-\mathrm{E}$ & $13.57 \mathrm{~B}-\mathrm{G}$ & $12.93 \mathrm{G}-\mathrm{L}$ & $13.79 \mathrm{~A}-\mathrm{C}$ \\
\hline Chitosan $1.5 \%$ & $14.33 \mathrm{~A}$ & 14.13 A-D & $13.77 \mathrm{~A}-\mathrm{F}$ & $13.33 \mathrm{E}-\mathrm{J}$ & $12.80 \mathrm{I}-\mathrm{M}$ & 13.67 B-D \\
\hline Hot water $44^{\circ} \mathrm{C}$ & $14.33 \mathrm{~A}$ & 14.10 A-D & $13.70 \mathrm{~A}-\mathrm{F}$ & $13.17 \mathrm{~F}-\mathrm{K}$ & $12.67 \mathrm{~J}-\mathrm{N}$ & $13.59 \mathrm{~B}-\mathrm{E}$ \\
\hline Hot water $44^{\circ} \mathrm{C}+$ Chitosan $1 \%$ & $14.33 \mathrm{~A}$ & $14.27 \mathrm{AB}$ & 14.20 A-C & $13.93 \mathrm{~A}-\mathrm{E}$ & $13.63 \mathrm{~A}-\mathrm{G}$ & $14.07 \mathrm{~A}$ \\
\hline Hot water $44^{\circ} \mathrm{C}+$ Chitosan $1.5 \%$ & $14.33 \mathrm{~A}$ & $14.23 \mathrm{~A}-\mathrm{C}$ & 14.00 A-E & $13.63 \mathrm{~A}-\mathrm{G}$ & $13.20 \mathrm{~F}-\mathrm{K}$ & $13.88 \mathrm{AB}$ \\
\hline Hot water $48^{\circ} \mathrm{C}$ & $14.33 \mathrm{~A}$ & 13.97 A-E & $13.53 \mathrm{C}-\mathrm{H}$ & $12.70 \mathrm{~J}-\mathrm{M}$ & $11.97 \mathrm{NO}$ & $13.30 \mathrm{EF}$ \\
\hline Hot water $48^{\circ} \mathrm{C}+$ Chitosan $1 \%$ & $14.33 \mathrm{~A}$ & $14.03 \mathrm{~A}-\mathrm{E}$ & $13.63 \mathrm{~A}-\mathrm{G}$ & $12.97 \mathrm{G}-\mathrm{L}$ & $12.43 \mathrm{~L}-\mathrm{O}$ & $13.48 \mathrm{C}-\mathrm{F}$ \\
\hline Hot water $48^{\circ} \mathrm{C}+$ Chitosan $1.5 \%$ & $14.33 \mathrm{~A}$ & $14.00 \mathrm{~A}-\mathrm{E}$ & $13.57 \mathrm{~B}-\mathrm{G}$ & $12.83 \mathrm{H}-\mathrm{L}$ & $12.10 \mathrm{M}-\mathrm{O}$ & $13.37 \mathrm{D}-\mathrm{F}$ \\
\hline Control & $14.33 \mathrm{~A}$ & 13.93 A-E & 13.43 D-I & 12.57 K-N & $11.77 \mathrm{O}$ & 13.21 F \\
\hline Mean & $14.33 \mathrm{~A}$ & $14.10 \mathrm{~B}$ & $13.75 \mathrm{C}$ & 13.19 D & $12.61 \mathrm{E}$ & \\
\hline
\end{tabular}

Means in the same column having the same letter are not significantly different at 0.05 level by Duncan's multiple rang test.

Regarding the effect of postharvest treatments, data revealed that artichoke heads dipped in hot water at $44^{\circ} \mathrm{C}$ plus chitosan $1 \%$, hot water at $44^{\circ} \mathrm{C}$ plus chitosan $1.5 \%$ and chitosan $1 \%$ alone were the most effective treatments in maintaining TSS with no significant differences between them followed by chitosan at $1.5 \%$ or hot water at $44^{\circ} \mathrm{C}$ with no significant differences between them. The lowest values resulted in other treatments and untreated control. These results were achieved in the two seasons and were in agreement with those obtained by Hong et al. (2012) and Shiri et al. (2013) for chitosan and Abdalla (2013) for hot water.

The effect of chitosan treatment on soluble solid contents was probably due to the slowing down of respiration and metabolic activity, hence retarding the ripening process, modifying the internal atmosphere by reducing $\mathrm{O}_{2}$ and/or elevating $\mathrm{CO}_{2}$ and suppressing ethylene evolution. The modified atmosphere created by chitosan coating suppresses the loss of TSS\% during storage (Yonemoto and Higuchi, 2002; Dong et al., 2004; Shiri et al., 2013). The higher level of TSS in the fruit coated with chitosan may be due to the reduction of the oxygen supply on the fruit surface, which reduced respiration, thus slowing the changes of TSS contents (Yonemoto and Higuchi, 2002).

The positive effect on total soluble solids by hot water treatment may be due to the delaying ripening of heated tomatoes, and related to the lower consumption of sugars as the result of the reduction in the ripening rate during storage following heat treatment (Lu et al., 2010).

In general, the interaction between postharvest treatments and storage periods was significant in the two seasons. After 28 days of storage, artichoke heads dipped in hot water at $44^{\circ} \mathrm{C}$ plus chitosan $1 \%$ and hot water at $44^{\circ} \mathrm{C}$ plus chitosan $1.5 \%$ resulted in 
higher total soluble solids with no significant differences between them, while untreated control, hot water at $48^{\circ} \mathrm{C}$ alone, hot water at $48^{\circ} \mathrm{C}+$ chitosan $1 \%$, hot water at $48^{\circ} \mathrm{C}$ plus chitosan $1.5 \%$ gave the lowest ones in the same period with no significant differences between them. These results were true in the two seasons and in agreement with those of Djioua et al. (2010) that found that hot water treatment plus chitosan coating resulted in higher TSS level than in control. Then, during the storage TSS level remained stable for every condition, demonstrating that neither hot water treatment nor chitosan coating have an effect on TSS of fresh-cut mangoes.

\section{Inulin content}

Data in Table (9) showed that inulin content of heads were significantly decreased with the prolongation of storage period. Similar results were obtained by Atala (2006). The loss of inulin content during the storage period may be attributed to respiration and other senescence related metabolic processes during storage (Wills et al., 1998).

Regarding the effect of postharvest treatments, data revealed that artichoke heads dipped in hot water at $44^{\circ} \mathrm{C}$ plus chitosan $1 \%$ and hot water at $44^{\circ}$ $\mathrm{C}$ plus chitosan $1.5 \%$ were the most effective treatments in maintaining inulin content with significant differences between them followed by chitosan at $1 \%$. However, hot water at $48^{\circ} \mathrm{C}$ plus chitosan $1 \%$ or hot water at $48^{\circ} \mathrm{C}$ plus chitosan $1.5 \%$ treatments were less effective in this concern. The lowest values resulted in untreated control. These results were achieved in the two seasons and were in agreement with those obtained by Haggag et al. (2017) for chitosan.

Table 9. Effect of hot water treatment and chitosan coating alone or in a combination between them on inulin content (g/100 gm d.w) of artichoke heads during cold storage.

\begin{tabular}{|c|c|c|c|c|c|c|}
\hline \multirow{3}{*}{ Treatments } & \multicolumn{5}{|c|}{ Storage period(days) } & \multirow{3}{*}{ Mean } \\
\hline & \multicolumn{5}{|c|}{2017 season } & \\
\hline & start & 7days & 14days & 21days & 28days & \\
\hline Chitosan 1\% & $15.62 \mathrm{~A}$ & 15.20 B-D & $14.40 \mathrm{H}$ & $14.00 \mathrm{IJ}$ & 13.20 LM & $14.48 \mathrm{C}$ \\
\hline Chitosan $1.5 \%$ & $15.62 \mathrm{~A}$ & $15.10 \mathrm{CD}$ & $14.00 \mathrm{IJ}$ & $13.50 \mathrm{~K}$ & $12.80 \mathrm{NO}$ & 14.20 D \\
\hline Hot water $44^{\circ} \mathrm{C}$ & $15.62 \mathrm{~A}$ & $15.00 \mathrm{DE}$ & $13.80 \mathrm{~J}$ & 13.20 LM & $12.50 \mathrm{P}$ & $14.02 \mathrm{E}$ \\
\hline Hot water $44^{\circ} \mathrm{C}+$ Chitosan $1 \%$ & $15.62 \mathrm{~A}$ & $15.40 \mathrm{AB}$ & $15.00 \mathrm{DE}$ & $14.80 \mathrm{EF}$ & $14.10 \mathrm{I}$ & $14.98 \mathrm{~A}$ \\
\hline Hot water $44^{\circ} \mathrm{C}+$ Chitosan $1.5 \%$ & $15.62 \mathrm{~A}$ & $15.30 \mathrm{BC}$ & $14.80 \mathrm{EF}$ & $14.40 \mathrm{H}$ & $13.80 \mathrm{~J}$ & $14.78 \mathrm{~B}$ \\
\hline Hot water $48^{\circ} \mathrm{C}$ & $15.62 \mathrm{~A}$ & $14.50 \mathrm{GH}$ & 13.20 LM & $12.40 \mathrm{P}$ & $11.50 \mathrm{~S}$ & $13.44 \mathrm{G}$ \\
\hline Hot water $48^{\circ} \mathrm{C}+$ Chitosan $1 \%$ & $15.62 \mathrm{~A}$ & $14.80 \mathrm{EF}$ & $13.50 \mathrm{~K}$ & $12.80 \mathrm{NO}$ & $11.80 \mathrm{R}$ & $13.70 \mathrm{~F}$ \\
\hline Hot water $48^{\circ} \mathrm{C}+$ Chitosan $1.5 \%$ & $15.62 \mathrm{~A}$ & $14.70 \mathrm{FG}$ & $13.40 \mathrm{KL}$ & $12.60 \mathrm{OP}$ & $11.60 \mathrm{RS}$ & $13.58 \mathrm{~F}$ \\
\hline Control & $15.62 \mathrm{~A}$ & $14.50 \mathrm{GH}$ & $13.00 \mathrm{MN}$ & $12.10 \mathrm{Q}$ & $11.00 \mathrm{~T}$ & 13.24 H \\
\hline Mean & $15.62 \mathrm{~A}$ & $14.94 \mathrm{~B}$ & $13.90 \mathrm{C}$ & 13.31 D & $12.48 \mathrm{E}$ & \\
\hline \multicolumn{7}{|c|}{2018 season } \\
\hline Chitosan 1\% & $15.77 \mathrm{~A}$ & $15.36 \mathrm{~B}-\mathrm{D}$ & $14.56 \mathrm{H}$ & $14.15 \mathrm{IJ}$ & 13.35 LM & $14.64 \mathrm{C}$ \\
\hline Chitosan $1.5 \%$ & $15.77 \mathrm{~A}$ & $15.26 \mathrm{CD}$ & $14.16 \mathrm{IJ}$ & $13.65 \mathrm{~K}$ & $12.95 \mathrm{NO}$ & $14.36 \mathrm{D}$ \\
\hline Hot water $44^{\circ} \mathrm{C}$ & $15.77 \mathrm{~A}$ & $15.16 \mathrm{DE}$ & $13.94 \mathrm{~J}$ & 13.35 LM & $12.65 \mathrm{P}$ & 14.17 E \\
\hline Hot water $44^{\circ} \mathrm{C}+$ Chitosan $1 \%$ & $15.77 \mathrm{~A}$ & $15.56 \mathrm{AB}$ & 15.14 D-F & $14.95 \mathrm{EF}$ & $14.25 \mathrm{I}$ & $15.13 \mathrm{~A}$ \\
\hline Hot water $44^{\circ} \mathrm{C}+$ Chitosan $1.5 \%$ & $15.77 \mathrm{~A}$ & $15.46 \mathrm{BC}$ & $14.94 \mathrm{E}-\mathrm{G}$ & $14.55 \mathrm{H}$ & $13.95 \mathrm{~J}$ & $14.93 \mathrm{~B}$ \\
\hline Hot water $48^{\circ} \mathrm{C}$ & $15.77 \mathrm{~A}$ & $14.66 \mathrm{GH}$ & $13.34 \mathrm{LM}$ & $12.55 \mathrm{P}$ & $11.65 \mathrm{~S}$ & $13.59 \mathrm{G}$ \\
\hline Hot water $48^{\circ} \mathrm{C}+$ Chitosan $1 \%$ & $15.77 \mathrm{~A}$ & $14.96 \mathrm{EF}$ & $13.64 \mathrm{~K}$ & $12.95 \mathrm{NO}$ & $11.95 \mathrm{R}$ & $13.85 \mathrm{~F}$ \\
\hline Hot water $48^{\circ} \mathrm{C}+$ Chitosan $1.5 \%$ & $15.77 \mathrm{~A}$ & $14.86 \mathrm{FG}$ & $13.54 \mathrm{KL}$ & $12.75 \mathrm{OP}$ & $11.75 \mathrm{RS}$ & $13.73 \mathrm{~F}$ \\
\hline Control & $15.77 \mathrm{~A}$ & $14.66 \mathrm{GH}$ & $13.14 \mathrm{MN}$ & $12.25 \mathrm{Q}$ & $11.15 \mathrm{~T}$ & $13.39 \mathrm{H}$ \\
\hline Mean & $15.77 \mathrm{~A}$ & $15.10 \mathrm{~B}$ & 14.04 C & $13.46 \mathrm{D}$ & $12.63 \mathrm{E}$ & \\
\hline
\end{tabular}

Means in the same column having the same letter are not significantly different at 0.05 level by Duncan's multiple rang test.

The reduction of inulin loss of heads during storage by using chitosan or hot water treatments may be attributed to these materials reduced the ethylene production by fruit thus respiration rate and enzymatic activity ( Trung et al., 2011) for chitosan; (Lu et al., 2010) for hot water; resulted in reducing consumption of inulin during storage.

In general, the interaction between postharvest treatments and storage periods was significant in the two seasons. After 28 days of storage, artichoke heads dipped in hot water at $44^{\circ} \mathrm{C}$ plus chitosan $1 \%$ and hot water at $44^{\circ} \mathrm{C}$ plus chitosan $1.5 \%$ resulted in higher inulin content with significant differences between them, while hot water at $48^{\circ} \mathrm{C}$ alone, hot water at $48^{\circ} \mathrm{C}+$ chitosan $1 \%$, hot water at $48^{\circ} \mathrm{C}$ plus chitosan $1.5 \%$ were less effective in this concern. Untreated control gave the lowest ones in the same period. These results were true in the two seasons.

\section{Conclusion}

Artichoke heads dipped in hot water at $44^{\circ} \mathrm{C}+$ chitosan $1 \%$ produced an improvement in market quality; it would reduce weight loss percentage, color change and maintained ascorbic acid content, TSS, 
dry matter and inulin content and did not show any change in general appearance after 28 days of storage at $0{ }^{\circ} \mathrm{C}$ and $95 \% \mathrm{RH}$.

\section{Reference}

Abdalla, M.A.A, 2013. Pre and Postharvest Treatments to Enhance Sweet Pepper (Capsicum annuum L.) Productivity and Quality. Ph.D. Thesis, Fac., Agric., Ain Shams Univ., Cairo, Egypt, $101 \mathrm{p}$.

Ahmed, H.H.A.; M.R. Aboul-Ella Nesiem; H.A. Allam and A.F. El-Wakil, 2016. Effect of preharvest chitosan foliar application on growth, yield and chemical composition of Washington navel orange trees grown in two different regions. Afri. J. of Bioche. Res., 10(7): 59-69.

Ardakani, M. D. and Y. Mostofi, 2019. Postharvest application of chitosan and Thymus essential oil increase quality of the table grape cv. 'Shahroudi'. J. of Horti. and Posth. Res., 2(1): 3142 .

Atala, S. A, 2006. Effect of some treatments for improving the quality and increasing storage ability of globe artichoke. M.Sc. Thesis, Fac., Agric.,Cairo Univ.,Egypt.

Chailoo, M. J. and M. R. Asghari , 2011. Hot water and chitosan treatment for the control of postharvest decay in sweet cherry (Prunus avium L.) cv. Napoleon (Napolyon) . J. of Stor. Prod. and Posth. Res., 2(7): 135-138.

Djioua,T.; F.Charles; M. Freire; H. Filgueiras; M.N. Ducamp- Collin and H.Sallanon, 2010. Combined effects of postharvest heat treatments and chitosan coating on quality of fresh-cut mangoes (Mangifera indica L.). Inter. J. of Food Sci. and Techn.,45: 849-855.

Dong,H.; L. Cheng; Tan and Y. Jiang, 2004. Effect of chitosan coating on quality and shelf-life of peeled litchi fruit. J. Food Eng.,64: 355-358.

El-Sayed, S.F.; M.A. El-Helaly; M.S. Emam and M.A. Abdel-Ghaffar, 2013. Effect of Some PostCold Storage Treatments on Shelf Life of Sweet Potato Roots. J. of Horti. Sci. and Orna. Plants, 5 (3): $160-170$.

Fallik, E. 2004. Prestorage hot water treatments (immersion, rinsing and brushing). Posth. Bio. and Technol., 32: 125-134.

Gemma, H. and J. Du, 1998. Effect of application of various chitosans with different molecular weights on the storability of "jonagold" apple. Acta Hortic., 464:536.

Gil-Izquierdo, A.; M. A. Conesa; F. Ferreres and M. I. Gil, 2002. Influence of modified atmosphere packaging on quality, vitamin $\mathrm{C}$ and phenolic content of artichokes (Cynara scolymus L.). Eur. Food Res. Technol., 215:21-27.

Gonzalez-Aguilar, G.; R. Cruz; R. Baez and C.Y. Wang, 1999. Storage quality of bell peppers pretreated with hot water and polyethylene packaging. J. Food Qual., 22(3):287-299.

Haggag, I.A.A.; S.A. Shanan; A.S.A.Abo El-Hamd ;A.A. Helaly and R.E.I. El-Bassiouny, 2017. Effect of temperature and modified atmosphere packaging on globe artichoke (Cynara scolymus L.) quality during storage. Adv. Plants Agric. Res., 6(5):138-145.

Hernandez-Munoz, P.; E.Almenar; V. Del Valle; D. Dinoraz Velez and R.Gavara, 2008. Effect of chitosan coating combined with postharvest calcium treatment on strawberry (Fragaria $\times$ ananassa) quality during refrigerated storage. Food Chemi., 110: 428-435.

Hirano, S.; Y. Ohe and H.Ono, 1976. Selective Nacylation of chitosan. Carbohyd. Res., 47: 315320.

Hong,K.Q.; J.H.Xie; L.B. Zhang; D.Q. Sun and D.Q. Gong, 2012. Effect of chitosan coating on postharvest life and quality of guava (Sodium guajava L.) fruit during cold storage. Scientia Horticultuae, 144:172-178.

Jianglian, S. and Z.Shaoying, 2013. Application of Chitosan Based Coating in Fruit and Vegetable Preservation. J. Food Process Technol., 4:227.

Jongsri, P.; T. Wangsomboondee; P.Rojsitthisak and K.Seraypheap, 2016. Effect of molecular weights of chitosan coating on postharvest quality and physicochemical characteristics of mango fruit. LWT - Food Sci. and Technol., 73: 28-36.

Kader, A. A. 1992. Postharvest biology and technology: an overview. In A.A. Kader (ed.). Postharvest technology of horticultural crops. California: University of California Puplications, P.15-20.

Kader, A. A.; L.L. Morris and E.C. Maxie, 1973. Systems for scoring quality of harvested lettuce. Hort. Sci., 8:408-409.

Lavermicocca , P.; M. Dekker; M. Russo; F. Valerio; F. Di Venere and D. Sisto, 2016. Lactobacillus paracasei-enriched vegetables containing health promoting molecules. In-R.R.Watson \& V.R. preedy (Eds.). Probiotics, prebiotics and synbiotics. Pioactive foods in health promotion. San Diego, CA, USA: Elsevier Academic press. (pp 361-370)

Lu,X. H.; D.Q. Sun; Y.W. J.C. Mo and G.M. Sun, 2010. Effect of post-harvest salicylic acid treatment on fruit quality and anti-oxidant metabolism in pineapple during cold storage. J. Hort. Sci. Biotechnol., 85:454 - 458 .

Mahmoud, T. Sh. M.; N. M. A. Yassin and F.K. M. Shaaban, 2017. Influence of Postharvest Application with Chitosan and some Natural Plant Extracts on Storage Life and Quality Attributes of Navel Orange Fruits during Cold Storage. Midd. East J. of Agric. Res., 6 (2):330339.

Mama, S.; J. Yemer and W.Woelore, 2016. Effect of Hot Water Treatments on Shelf Life of Tomato 
(Lycopersicon esculentum Mill). J. of Nat. Sci. Res., 6 (17): 69-77.

Mileo, A. M.; D. Di Venere; V. Linsalata; R. Fraioli and S. Miccadei, 2012. Artichoke polyphenoles induce apoptosis and decrease the invasive potential the human breast cancer line MDAMB231. J. of cellu. Phys., 227:3301-3309.

Mohamedien, S.E.A.; M.EL-M. Saad; S.A. Atala and E.H.E. Afifi, 2019. Effect of cooling delays on quality attribute of globe artichoke during cold storage. Annals of Agric. Sci., Moshtohor, 57(1):105-112.

Nobile, M. A.; A. Conte; C. Scrocco; J. Laverse; I. Brescia; G. Conversa and A. Elia, 2009. New packaging strategies to preserve fresh-cut artichoke quality during refrigerated storage. Innov. Food Sci. and Emer. Technol., 10: 128133.

Özden, Ç. and L. Bayindirli, 2002. Effects of combinational use of controlled atmosphere: Cold storage and edible coating applications on shelf life and quality attributes of green peppers. Euro. Food Res. and Technol., 214: 320-326.

Picha, D.H. 1987. Chilling injury, respiration and sugar changes in sweet potatoes stored at low temperatures. J. of the Amer. Soci. of Horti. Sci., 112(3): 497-502.

Ramos-García, M.; S.Ortega-Centeno; A.N. Hernández-Lauzardo; I.Alia-Tejacal; E.BosquezMolina and S. Bautista-Baños, 2009. Response of gladiolus (Gladiolus spp) plants after exposure corms to chitosan and hot water treatments. Sci. Horti., 121: 480-484.

Ranganna, S. 1979. Manual of analysis of fruit and vegetable products. Central Food Technological Research Institute. Oh.D. Published 12/4Asaf Ali ROAD,New Delhi 110002, and printed at raj Bandhu Industrial Company, C-16, May a puri, phase 11, New Delhi, 1100064.

Raymond, L.V.; M.Zhang and S.M.Roknul Azam, 2012. Effect of chitosan coating on physical and microbical characteristics of fresh-cut green peppers (Capsicum annum L.). Pakis. J. of nut. , 11 (10):806-811.

Rinaudo, M. 2006. Chitin and chitosan: properties and applications. Prog. Polym. Sci., 31:603-632.

Sakaldas, M. and K. Kaynas, 2010. Biochemical and quality parameters changes of green sweet bell peppers as affected by different postharvest treatments. J. of Biot., 9(48):8174-8181.

Shao, X.F.; K. Tu; S. Tu and J. Tu, 2012. A combination of heat treatment and chitosan coating delays ripening and reduces decay in "Gala" apple fruit. J. of Food Quali., 35: 83-92.

Shehata, S.A.; M.I.A. Ibrahim; M.M. El-Mogy and K.F. Abd El-Gawad, 2013. Effect of hot water dips and modified atmosphere packaging on extend the shelf life of bell pepper fruits. Wulf. J., 20 (3): 315-328.
Shiri,M.A.;D.Bakhshi;M.Ghasemnezhad;M.Dadi; A.Papachatzism and H. Kalorizou, 2013. Chitosan coating improves the shelf life and postharvest quality of table grape (Vitis vinifera) cultivar Shahroudi. Turk J. Agric., 37:148-156.

Snedecor, G. W. and W. G. Cochran, 1980.Statistical methods. $6^{\text {th }}$ Iowa State Univ. Press, USA.

Suseno, N.; E.Savitri; L.Sapei and K. S. Padmawijaya, 2014. Improving shelf-life of 'Cavendish' banana using chitosan edible coating. Procedia Chemistry, 9: 113-120.

Trung, T.S.; N.T. Hang Phuong and W.F. Stevens, 2011. Protective effect of chitosan coating and polyethylene film wrapping on postharvest storage of sugar-apples. As. J.Food Ag-Ind., 4(2):81-90.

Vargas, M.; A. Albors; A. Chiralt and C. GonzalezMartinez , 2006. Quality of cold stored strawberries as affected by chitosan-oleic acid edible coatings. Postharvest Biol. Technol., 41: 164-171.

Velickova, E.; E. Winkelhausen; S. Kuzmanova; V.D. Alves and M .Moldao-Martins, 2013. Impact of chitosan-beeswax edible coatings on the quality of fresh strawberries (Fragaria ananassa cv Camarosa) under commercial storage conditions. LWT-Food Sci. Technol., 52: 80-92.

Waller, R. A. and D. B. Duncan, 1969. A buyes rule for the symmetric multiple comparison problems. Amer. State. Assoc. J., 64:1484-1503.

Wills, R.; B. Mc Glasson; D. Graham and D. Joyce, 1998.Postharvest: An introduction to the physiology and Handling of fruit, vegetables and ornamentals ( $4^{\text {th }}$ end $\left.n\right) . C A B$ international, New York, PP. 77-96.

Winton, A.L. and K.B. Winton, 1958. The Analysis of Foods. John Wiley and Sons. Inc. London.pp.857.

Xu, W.T.; K. 1. Huang; F. Guo; W. Qu; J.J. Yang; Z.H.Liang and Y. B. Luo, 2007. Postharvest grapefruit seed extract and chitosan treatments of table grapes to control Botrytis cinerea. Posth. Bio. and Technol., 46:86-94.

Yonemoto, Y. and H.K. Higuchi, 2002. Effect of storage temperature and coating on ethylene production, respiration and Shelf-Life in cherimoya fruit. Japanese Soc. for Hort. Sci., 71: 643-650.

Youssef, A.R.M.; E.A.M. Ali and H.E. Emam, 2015. Influence of postharvest applications of some edible coatings on storage life and quality attributes of Navel orange fruit during cold storage. Int. J. Chem. Tech. Res., 8(4): 21892200 .

Zahoorullah, S. M.; L. Dakshayani; A. S. Rani and G. Venkateswerlu, 2017. Effect of chitosan coating on the post-harvest quality of banana during storage. Asian J. of Biot. and Biores. Technol., 1: 1-10. 
Zhao, Y.; K.Tu; J.Su; S. Tu; Y.Hu; F. Lu and X. $\mathrm{Zu}, 2009$. Heat treatment in combination with antagonistic yeast reduces diseases and elicits the active defense responses in harvested cherry tomato fruit. J.Agric. Food Chem., 57:75657570 .

\section{تأثير استخدام معاملات الماء الساخن و الثبتوسان لتحسين صفات الجودة وزيادة القدرة التخزينية لنورات الخرشوف. شيرين عطا عطالله, محسن السيد محمد سعد, محمود عاطف صالح. معهد بحوث البساتين -مركز البحوث الزراعية بالجيزة.}

تم جمع نورات الخرشوف صنف French Hyrious فى مرحلة القطف المناسبة للتسويق فى 5 ، 9 فبراير موسمى 2017 ، 2018 على التوالى من مزرعة خاصة في كفر الدوار - سيدي غازي - محافظة البحيرة ثم نقلت الى معمل بحوث تداول الخضر - معهد بحوث البساتين

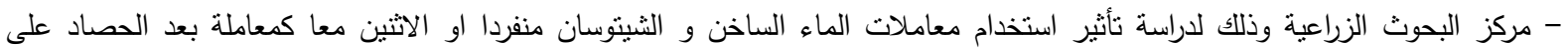
صفات الجودة لنورات الخرشوف اثثاء التخزين المبرد. أوضحت النتائج ان جميع معاملات ما بعد الحصاد ادت الى بطىء معدل الفقد فى الوزن لنورات الخرشوف ، و و كانت اكثر اندماجا و

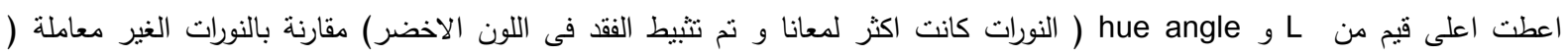

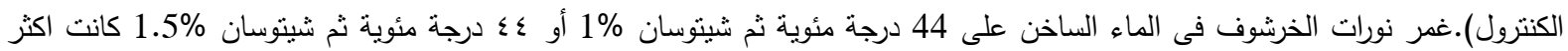

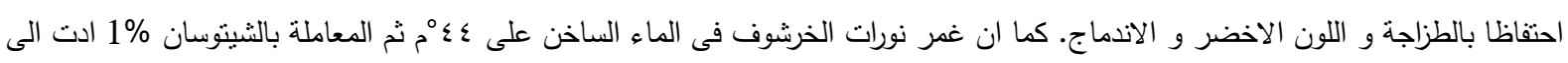
تحسين الجودة التسويقية ، حيث قللت من فقد الوزن ، الفقد فى اللون كما احتفظت بحمض الاسكوربيك و المواد الصلبة الذائبة و المادة الجافة

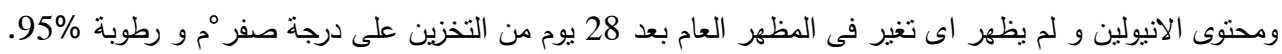

